\title{
On the merger of subsurface isolated vortices
}

\author{
Ciani Daniele ${ }^{1,{ }^{*}}$, Carton Xavier ${ }^{1}$, Verron Jacques ${ }^{2}$
}

${ }^{1}$ CNRS Ifremer IRD UBO, Lab Phys Oceans, UMR6523, Brest, France.

${ }^{2}$ CNRS UJFR, Lab Glaciol \& Geophys Environm, UMR5183, Grenoble, France.

*Corresponding author : Daniele Ciani, email address : daniele.ciani@univ-brest.fr

\begin{abstract}
:
Vortex merger is a phenomenon characterizing the whole class of geophysical vortices, from atmospheric storms and large oceanic eddies up to small scale turbulence. Here we focus on the merger of subsurface oceanic anticyclones in an idealized primitive equations model. This study has been motivated by past and recent observations of colliding lens-like anticyclones off of Gibraltar Strait. The critical conditions for merger (critical merger distance and time needed for merger) are determined. We will show that the predictions of classical two-dimensional merger are not verified for subsurface isolated vortices. For instance, critical merger distances will be reduced because of the vortex potential vorticity (PV) structure. The post-merger characteristics of the vortex (radius, extension and PV), are also determined. Merger-related effects, like production of peripheral filaments and small-scale eddies are also investigated and suggest the contribution of merger in both direct and inverse energy cascades.
\end{abstract}

Keywords : subsurface isolated vortices, Peddies, Meddies, Vortex merger

\section{Introduction}

The global ocean is highly populated by vortical structures. Such features are recirculating motions at both mesoscale and submesoscale; they can be found at di_erent depths in the water column (Ebbesmeyer et al. 1986, Carton 2001, Chelton et al. 2011, Shapiro and Meschanov 1991, Richardson et al. 2000, Rubino et al. 2012, Ciani et al. 2015). The mechanisms generating such structures range from the instability of large scale currents to small scale turbulence or deep convection. Due to planetary rotation and to the oceanic stable strati_cation, mesoscale vortex motions are mostly con_ned in the horizontal plane. Thus, vortices have a major impact on the evolution of water masses across the oceans, regulating the three-dimensional distribution of active and passive oceanic tracers.

Oceanic vortices often interact with currents, topography or other vortices (Masina and Pinardi 1991, Schultz Tokos et al. 1994, Cenedese et al. 2005, Filyushkin et al. 2011, Bar- bosa Aguiar et al. 2013, Sokolovskiy et al. 2013, L'H_egaret et al. 2014, Molemaker et al. 2014). Among these interactions, vortex merger gives rise to larger structures; it has been shown in geophysical turbulence studies to explain the inverse energy cascade (i.e., energy 
transfer from small to larger spatial scales). Merger happens when two like-signed vortices get in contact, start exchanging fluid masses, collapse towards each other and eventually generate a single coherent structure. The merger of vortical structures was also considered in atmospheric studies and engineering applications, since it plays a role in the evolution of air masses (Kieu and Zhang 2008) and in the dynamics of propeller wakes or wind turbines (Delbende et al. 2015).

In an oceanographic context, vortex merger has been mostly investigated and observed by means of in-situ measurements (Schultz Tokos et al. 1994, L'Hégaret et al. 2014), realistic modeling (Barbosa Aguiar et al. 2013, Molemaker et al. 2014), and analytical and idealized numerical modeling in a quasi-geostrophic context (Carton 1992, Masina and Pinardi 1991, Verron et al. 1990, Reinaud and Dritschel 2005, Bambrey et al. 2007). Potential Vorticity (PV) or its anomaly with respect to rest, is a key quantity to study the problem, since it is conserved for all fluid elements in absence of dissipation.

In past studies, it has been pointed out that critical conditions have to be verified for merger to occur. For instance, the distance between the two vortices has to reach a threshold which depends on the vortex potential vorticity structure.

When barotropic vortices are studied, provided a uniform PV structure (unshielded vortex), this distance (evaluated between the centers) is about 3.2 to $3.3 R$, where $R$ is the vortex radius (Overman et al. 1981, Dritschel 1985, Dritschel and Legras 1991, Dritschel 1995). If more realistic vortices are studied (e.g. Gulf-Stream rings) the critical distance can be smaller than 2.5 R , as stated by Masina and Pinardi (1991) and Valcke and Verron (1997). For realistic vortices, the PV structure changes in sign in the horizontal or in the vertical (Olson 1980 , Paillet et al. 2002). In the first case, the horizontal PV will be composed of a negative core surrounded by an opposite sign annulus. This annulus can reduce the critical merging distance due to shielding effect of the vorticity annulus (Carton 1992).

On the other hand, Reinaud and Dritschel (2005) pointed out that, in three dimensional contexts, the merging process is also influenced by the vortex shape. Prolate and tilted structures can in fact become unstable and eventually merge at higher distances than predicted by classical merger. Moreover, Filyushkin and Sokolovskiy (2011) have shown that the critical distance for the merging lenses (which are supposed to be vortex patches in the middle layer in the three-layer quasi-geostrophic model) is a non-monotonous function of the Froude number.

In this work, we investigate the merger of two identical subsurface anticyclones in the framework of the full primitive equations, for idealized vortices representing several types of oceanic subsurface eddies, e.g., Meddies, Peddies and Reddies, respectively subsurface eddies generated by the Mediterranean water, Persian Gulf water and Red Sea water outflows (Richardson et al. 2000, Senjyu et al. 1998, Shapiro and Meschanov 1991). This study is carried out by means of the Regional Oceanic Modeling System (ROMS, Shchepetkin and McWilliams 2005). The study has been motivated by occurrence of Meddy collisions off of Gibraltar strait (Schultz Tokos et al. 1994, Barbosa Aguiar et al. 2013, L'Hégaret et al. 2014) as well as the detection of small lens-like vortices (radius $\simeq 15 \mathrm{~km}$ ), the so called Peddies, generated by the Persian Gulf water outflow in the Arabian Sea (L'Hégaret 2015). We will summarize the family of subsurface eddies using two types of idealized structures, that will be labelled with 
$\mathrm{M}$ and $\mathrm{P}$, to indicate larger Meddy-like vortices and the smaller Peddy-like structures.

Here, the study will be carried out in an idealized context, which is the ideal framework for a parametric investigation. The subsurface anticyclones are here horizontally and vertically shielded. We will determine the critical conditions for merger of subsurface anticyclones to occur, also accounting for the sensitivity to vortex rotational velocity and to background stratification.

The paper is organized as follows. Section 2 focuses on the description of the model and the determination of the initial states for the merger experiments. We go on illustrating the results of the simulations in section 3 , defining the critical conditions for merger to take place. In section 4, we describe the effects of merger on the structure of the final vortex and in particular on its deformation. Finally, the conclusions are presented in section 5 .

\section{Model and Setting}

We study the interaction of subsurface anticyclones in an idealized flat-bottomed, doublyperiodic oceanic basin and in $f$-plane approximation. Our tool of investigation is the primitive equation ROMS model (Shchepetkin and McWilliams 2005). The oceanic basins under consideration are of three types, and they are chosen in order to fit the idealized structures investigated here. If larger Meddy-like vortices are dealt with (radius $\simeq 30 \mathrm{~km}$ ), the basin measures $500 \mathrm{~km} \times 500 \mathrm{~km} \times 3 \mathrm{~km}$ for the zonal, meridional and vertical coordinate, respectively. In this case, the horizontal resolution is $2 \mathrm{~km}$. For the study of Peddy-like structures (radius $\simeq 15 \mathrm{~km}$ ) we reduce the horizontal extension of the basin to $300 \mathrm{~km} \times 300 \mathrm{~km}$ but we increase the horizontal resolution to $1 \mathrm{~km}$. In a final study, in order to describe smaller features generated after the merger, we keep the horizontal extension of the basin to $300 \mathrm{~km} \times 300 \mathrm{~km}$ and we choose a horizontal resolution of $500 \mathrm{~m}$. For all the cases, the vertical discretization is given by 80 non-uniformly spaced levels (a lower resolution is set for bottom levels). All the experiments are characterized by a free decaying dynamics. Each of the analyzed simulations is initialized by means of a background density stratification

$$
\bar{\rho}(z)=\rho_{0} \exp (-\alpha z / \bar{H})
$$

with $\alpha=0.18, \bar{H}=3 \mathrm{~km}$ (the vertical extension of the basin) and $\rho_{0}=1027.5 \mathrm{~kg} \cdot \mathrm{m}^{-3}$. The parameters have been chosen in order to respect the geography of the Rossby radius of deformation $\left(R_{d}\right.$ hereinafter) (Chelton et al. 1998). We initially set this parameter to about $36 \mathrm{~km}$, which is a typical value of $R_{d}$ in the north-eastern Atlantic. Furthermore, such a $R_{d}$ is a good approximation of the internal radius of deformation in the northern Arabian Sea (about $40 \mathrm{~km}$ ), making it suitable for both Meddies and Peddies/Reddies applications. This choice is justified by occurrences of Meddy collisions described by numerical models and observations (Schultz Tokos et al. 1994, Barbosa Aguiar et al. 2013, L'Hégaret et al. 2014). Note that $R_{d}$ is computed via determination of the baroclinic modes associated to our analytic stratification and it is the internal radius of deformation related to the first baroclinic mode.

Cyclogeostrophic balance is used in order to derive the density anomaly associated to the subsurface anticyclones at the beginning of the simulation. In this way, the Coriolis accelera- 
tion, pressure gradient field and the centrifugal acceleration can be taken into account, which is an advisable choice if structures with $R o \simeq 1$ are studied (Carton 2001).

In cylindrical coordinates, the cyclogeostrophic balance is expressed by

$$
\frac{v_{\theta}^{2}}{r}+f_{0} v_{\theta}=\frac{1}{\rho_{0}} \frac{\mathrm{d} p}{\mathrm{~d} r}(r, z)
$$

with $r$ representing the distance from the center of a generic vortex, $v_{\theta}$ its azimuthal velocity, $f_{0}$ the Coriolis parameter and $\mathrm{d} p / \mathrm{d} r$ the pressure gradient field. This relation, provided knowledge of the velocity field, contains the information on the density structure associated to the vortex. This balance is only an approximation of the gradient-wind balance, which should be used for multiple vortices, but which is more complex to implement. The initial velocity field is an analytical approximation of a subsurface anticyclonic vortex:

$$
v_{\theta}=-\frac{v_{0} r}{R} \exp \left(-\frac{r^{2}}{R^{2}}\right) \exp \left(-\frac{\left(z-z_{0}\right)^{2}}{H^{2}}\right)
$$

where $v_{0}$ is the intensity of the field (in $\mathrm{rad} \cdot \mathrm{s}^{-1}$ ), $R$ its Radius, $z_{0}$ its position along the vertical and $H$ the thickness. Integrating (2) between $r$ and $\infty$, and, noting that only coherent vortices are treated in this work, the pressure field can be assumed to be zero far from the vortex center (namely at $r=\infty$ ) so that one can write

$$
\int_{r}^{\infty} \frac{\mathrm{d} p^{\prime}}{\mathrm{d} r^{\prime}}\left(r^{\prime}, z\right) \mathrm{d} r^{\prime}=p^{\prime}(\infty)-p^{\prime}(r, z)=-p^{\prime}(r, z)
$$

which finally provides the pressure field

$$
p^{\prime}(r, z)=\beta \exp \left(-\frac{2\left(z-z_{0}\right)^{2}}{H^{2}}\right) \exp \left(-\frac{2 r^{2}}{R^{2}}\right)-\gamma \exp \left(-\frac{\left(z-z_{0}\right)^{2}}{H^{2}}\right) \exp \left(-\frac{r^{2}}{R^{2}}\right)
$$

with

$$
\beta=\frac{\rho_{0} v_{0}^{2}}{4}, \quad \gamma=\frac{\rho_{0} f_{0} v_{0} R}{2} .
$$

The $z$-derivative of (5) yields the density anomaly associated with the vortex and this anomaly is then superposed to the rest stratification to obtain the total three-dimensional field. The hydrostatic equilibrium is imposed and it prescribes density anomalies directly from pressure anomaly $p^{\prime}(r, z)$ :

$$
\frac{1}{g} \frac{\mathrm{d} p^{\prime}}{\mathrm{d} z}(r, z)=-\rho^{\prime}(r, z)
$$

where $g$ is the gravity acceleration. A snapshot of a typical initial state for subsurface anticyclones merger experiments is shown in figure 1. The vertical sections of density, velocity field and Ertel potential vorticity anomaly (PVa hereinafter) are given for Meddy-like (M) and Peddy-like (P) vortices (hereinafter, the $\mathrm{M}$ and $\mathrm{P}$ label will be used for simplicity). Note that the expression for the Ertel potential vorticity is given by

$$
P V=\frac{1}{\rho}\left[\left(\partial_{x} v-\partial_{y} u+f\right) \partial_{z} \rho-\partial_{z} v \partial_{x} \rho+\partial_{z} u \partial_{y} \rho\right]
$$

with $(u, v)$ the zonal and meridional components of the velocity field, respectively (Vallis 2006). The PVa is computed from (7) as

$$
P V a(\bar{x}, t)=P V(\bar{x}, t)-P V_{\text {rest }}(\bar{x}, t)
$$


where $\bar{x}=(x, y, z)$ and the subscript rest indicates a region which is not affected by the eddies' dynamics.

These initial states represent our reference configuration, as they fit most of the observed subsurface vortices in the world ocean, (Pingree and Le Cann 1993, Carton 2001, Bashmachnikov and Carton 2012).
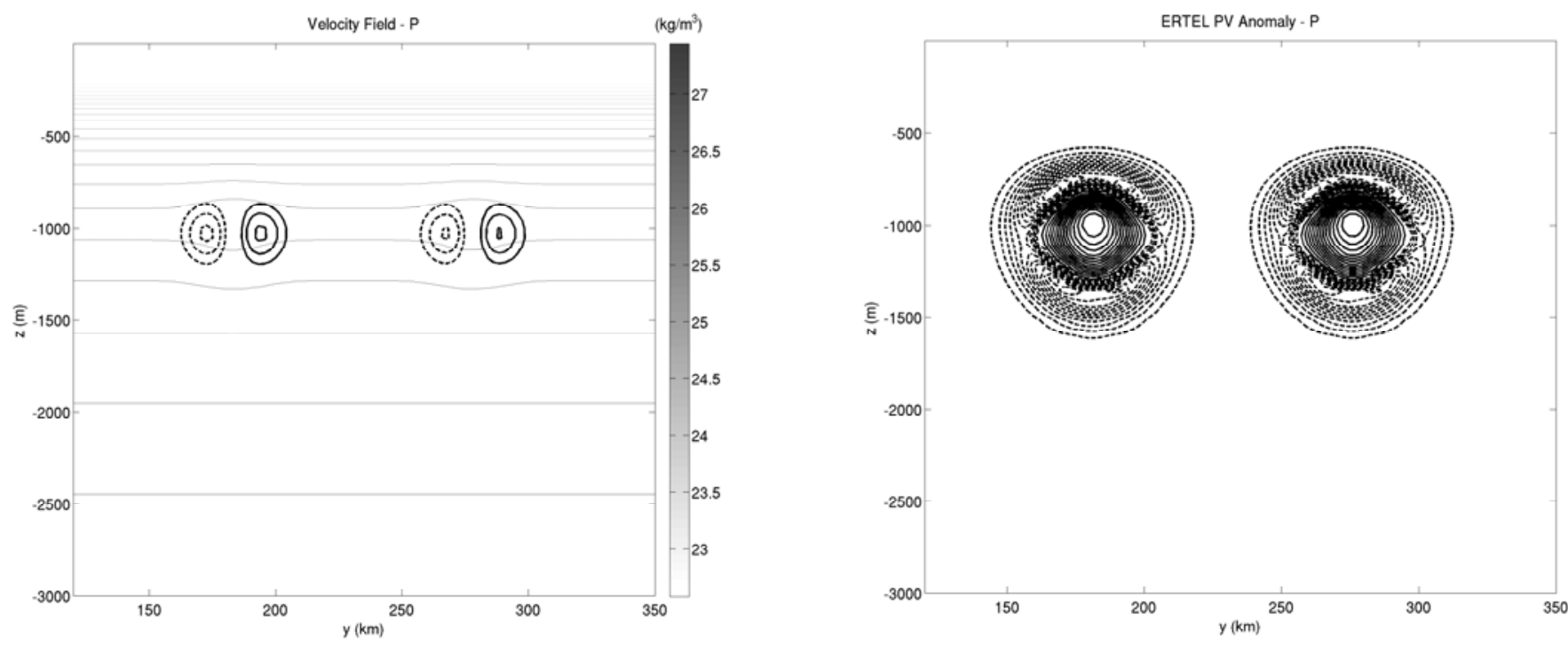

4
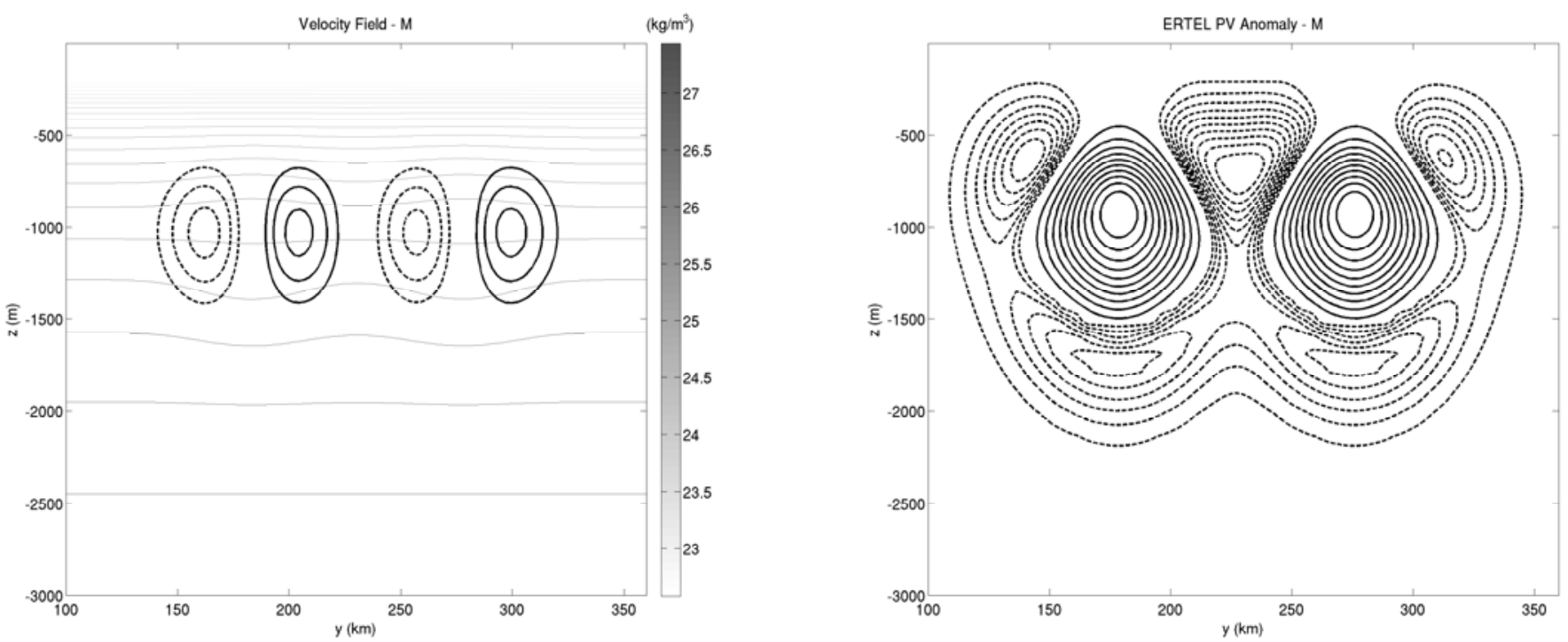

Figure 1. Initial state for subsurface vortex merger experiments with the ROMS model. Top-left and bottom-left: Vertical sections of density and swirl velocity field for $\mathrm{P}$ and $\mathrm{M}$-vortices. The bold contours indicate the zonal component of the swirl velocity and go from $0.1 \mathrm{~m} / \mathrm{s}$ to $0.2 \mathrm{~m} / \mathrm{s}$ at intervals of $0.05 \mathrm{~m} / \mathrm{s}$. Dashed and continuous lines indicate a flow directed towards or going out of the $y-z$ plane, respectively. Top-right and bottom-right: Vertical section of PVa for $\mathrm{P}$ and M-vortices. The PVa shields are given by dashed contours.

The PVa vertical structure at time $t=0$, figure 1 ( $\mathrm{M}$ and $\mathrm{P}$ case), suggests that we are dealing with a hybrid structure containing features of both $S$-vortices and $R$-vortices. $S$ and $R$-vortices are idealized quasi-geostrophic interior eddies whose potential vorticity structure (QGPV) is determined by the relative dominance of stretching and rotation, respectively 
(Morel and McWilliams 1997). In the $R$ case, the eddy exhibits a negative QGPV core surrounded by an opposite sign QGPV annulus, while $S$ structures consist of a QGPV negative core vertically bounded by opposite sign QGPV lobes (see also figure 1 in Morel and McWilliams 1997). In our case, a negative PVa core is horizontally and vertically shielded by means of a positive PVa annulus and a pair of positive lobes, respectively. If the relative distance between the two anticyclones decreases, the positive PVa annuli, surrounding the vortex internal cores can interact, thus generating a series of alternating positive and negative PVa poles, as shown in the bottom-right panel of figure 1. In the same panel, we show that the PVa upper lobes are absent. This is due to the steepness of the chosen density profile (exponentially decaying with depth, as shown in equation 1) close to the sea-surface. For shallower and deeper eddies (top-right panel of figure 1) both the horizontal and vertical shields are present.

As also illustrated by earlier works (Carton 1992, Valcke and Verron 1997), we will show the role played by the PVa annuli in the merger, weakening its efficiency via dipolar effects.

In the P case, the pair of anticyclones is initially placed at a depth of $1000 \mathrm{~m}$, the vortex radii are of $15 \mathrm{~km}$, their thickness is around $200 \mathrm{~m}$ and the initial rotational speed is of $0.2 \mathrm{~m} / \mathrm{s}$. For the M pair, depth and rotational velocities remain the same, and we choose a radius of $30 \mathrm{~km}$ and a thickness around $500 \mathrm{~m}$. This can be justified by observations of subsurface anticyclones in the mid-latitude ocean (Pingree and Le Cann 1993, Carton 2001, Bashmachnikov and Carton 2012). The dimensionless parameters describing our problem are the Rossby number $R_{0}$, the Burger number $B u$, the Reynolds number $R e$ and the stretching term $S$. They are all summarized in table 1 and, hereinafter, they will also be used for the characterization of subsurface vortices. Note that in table 1, we introduce three quantities:

- $\nu$, the fluid kinematic viscosity;

- $\Delta h$, the vortex induced isopycnal deformation, evaluated along the vertical axis, at the level where $\partial_{y} \rho(y, z)$ exhibits its maximum value (solid arrow in figure 2).

- $\bar{h}$, the thickness of the unperturbed isopycnal layer bounding the subsurface vortex (evaluated at $r=\infty$ from the vortex center, dashed arrow in figure 2);

Table 1. Characteristics of the modelled subsurface vortices in terms of dimensionless hydrodynamical numbers.

\begin{tabular}{cccc}
\hline & Definition & Value $(\mathrm{M})$ & Value $(\mathrm{P})$ \\
\hline BURGER NUMBER & $B u=\left(R_{d} / R\right)^{2}$ & 1.36 & 5.42 \\
REYNOLDS NUMBER & $R e=v_{\theta} R / \nu$ & $\gg 1$ & $\gg 1$ \\
ROSSBY NUMBER & $R_{0}=v_{\theta} /\left(f_{0} R\right)$ & 0.13 & 0.26 \\
STRETCHING FACTOR & $S=(\Delta h-\bar{h}) / \bar{h}$ & 0.35 & 0.41 \\
\hline
\end{tabular}

Summarizing, the subsurface vortex merger experiments take place in a high $R e$ regime. Though explicit kinematic viscosity is set to zero in our experiments, $R e$ is not infinite. Indeed, the model advection scheme introduces an intrinsic (numerical) viscosity. Furthermore, if rotational velocities are set to their reference value, geostrophy is best verified for $\mathrm{M}$ vortices $(R o \ll 1)$. The stretching term indicates that in both $\mathrm{M}$ and $\mathrm{P}$ cases, the relative isopycnal deformation is around $30 \%$. In the end, the Burger numbers suggest that, for the M case, stratification and rotation will influence the motion to a similar degree $(B u \simeq 1)$. In the $\mathrm{P}$ 


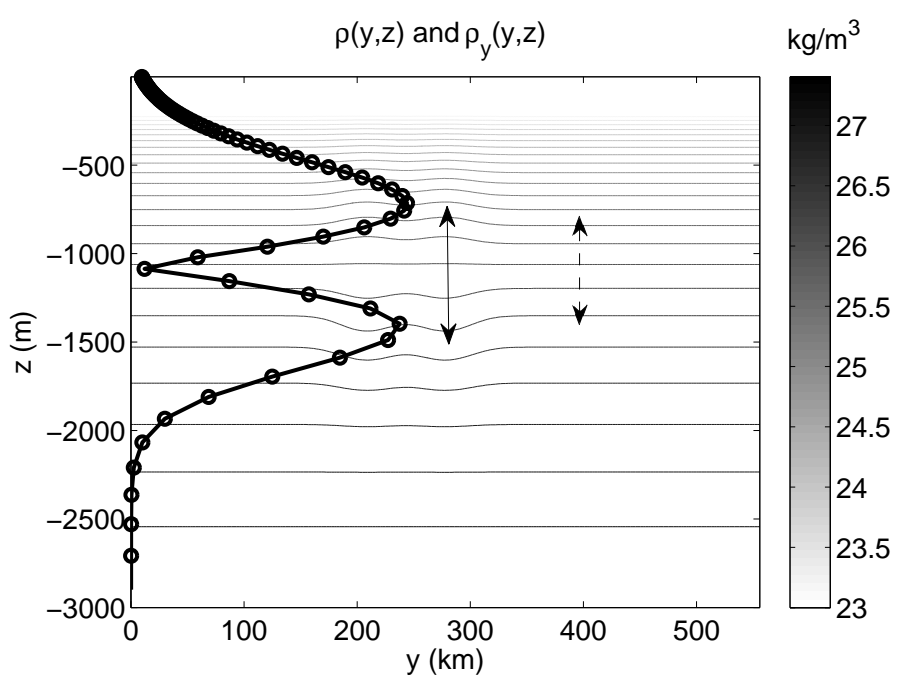

Figure 2. Meridional section of density field $\rho(y, z)$ and its meridional gradient (bold line), in a M case. The solid and dashed arrows indicate the isopycnal deformation induced by the subsurface vortex and the corresponding unperturbed isopycnal layer, respectively.

case $(B u>1)$, the effects of rotation will dominate and the dynamics will tend to a twodimensional-like behavior (Cushman-Roisin and Beckers 2011).

\section{Results}

We test the subsurface vortex merger sensitivity to the choice of initial parameters. This will allow us to determine the conditions under which this phenomenon can take place in an idealized primitive equations context. Our tool of investigation is the Regional Oceanic Modeling System (ROMS, Shchepetkin and McWilliams 2005).In particular, the occurrence of merger is studied as a function of vortices relative distance, initial rotational velocity and background stratification for both $\mathrm{M}$ and $\mathrm{P}$ structures. Such a parametric investigation will be achieved modifying the initial state parameters.

\section{1. $\quad$ Merger sensitivity to vortex parameters}

\subsubsection{Relative Distance}

We define the initial relative distance between the subsurface anticyclones as a function of the vortex radius $R$. In the $\mathrm{M}$ case, and for our potential vorticity distribution, the critical distance for merging occurrence $d_{c M}$ was found to be

$$
d_{c M}=2.5 R .
$$

An increase of this parameter of only about $0.1 R$ prevents the merging process to occur. This is shown by figure 3 and 4 , both illustrating the time evolution of a horizontal section of PVa at a depth of $1000 \mathrm{~m}$.

At the critical distance, the positive annuli interact, but are torn apart by the strong shear created by the cores. These two cores can collapse at the center, thus initializing the merging process (figure 3). The annuli are strongly deformed and advected around the central core. 
The filamentation of the strongly elongated core (at 12-14 days) leads to the injection of negative PVa into the annulus, which further weakens its influence. Finally, the central core has gathered the PVa of the two initial cores. For initially more distant vortices (figure 4) the annulus influence on the cores becomes stronger than the cores mutual interaction. The latter are advected radially outwards by their annulus via a dipolar effect and merger is prevented. This behavior is in agreement with the results of Valcke and Verron (1997), who, studying the merger of baroclinic shielded quasi-geostrophic vortices (with a potential vorticity structure comparable to that of an R-vortex), could determine two particular regimes of interaction: the Diverging and the Diverging Interaction case. In both cases, the merging process was not observed. This was due to a redistribution of the annuli's opposite-sign potential vorticity. Such a mechanism generates a repulsive effect on the two cores, thereby preventing their merger into a single core structure.

For $\mathrm{P}$ vortices, the critical initial distance for merger occurrence is of the same order of magnitude than in the $\mathrm{M}$ case, being

$$
d_{c P}=2.6 R \text {. }
$$

Furthermore, similarly to the previous case, an increase of this parameter of about $0.1 R$ prevents the merging process to take place. This is shown by figure 5 and 6 . These results show that the merger critical distance is smaller than the predictions of classical two-dimensional merger or of barotropic quasi-geostrophic vortices. This is in agreement with the results of Masina and Pinardi (1991) and Valcke and Verron (1997), who also found critical distances around $2.4 R$ for shielded baroclinic vortices by means of numerical experiments in the quasigeostrophic context.

\subsubsection{Influence of initial rotational velocity}

The effects of initial rotational velocity are investigated here. Choosing initial states already illustrated in figure 1 and Table 1, we let the initial rotational velocity vary from $0.1 \mathrm{~m} / \mathrm{s}$ to $0.4 \mathrm{~m} / \mathrm{s}$ and we observe the corresponding merging behavior. The dimensionless numbers affected by the changing rotational velocities, in the non-viscous limit, will be the Rossby number and the Stretching factor. The corresponding values for different experiments are summarized in Table 2. Such values indicate that increasing the intensity of the velocity field one gets less geostrophic vortices, as expressed by the increase of Rossby number. Moreover, equation 3 states that the velocity field determines the density anomaly associated to the vortex. Hence, the Stretching factor also varies when faster rotating vortices are dealt with. Indeed, if we increase the intensity of the velocity field without varying its horizontal extension, the vortex core isopycnal layers will be more stretched in the vertical, and the corresponding stretching factor will be larger.

In order to give a quantitative description of this process, we define the merging time as the time when the core of the final vortex (generated by the merger of the two initial subsurface anticyclones) is constituted by a single contour of PVa (where PVa contours are drawn from the minimum up the maximum PVa value, whose order of magnitude is $10^{-11} \mathrm{~m} / \mathrm{s}$, at intervals of $10^{-12} \mathrm{~m} / \mathrm{s}$ ). Note that this criterion for evaluating the merging time will also be used in section 3.1 .3 

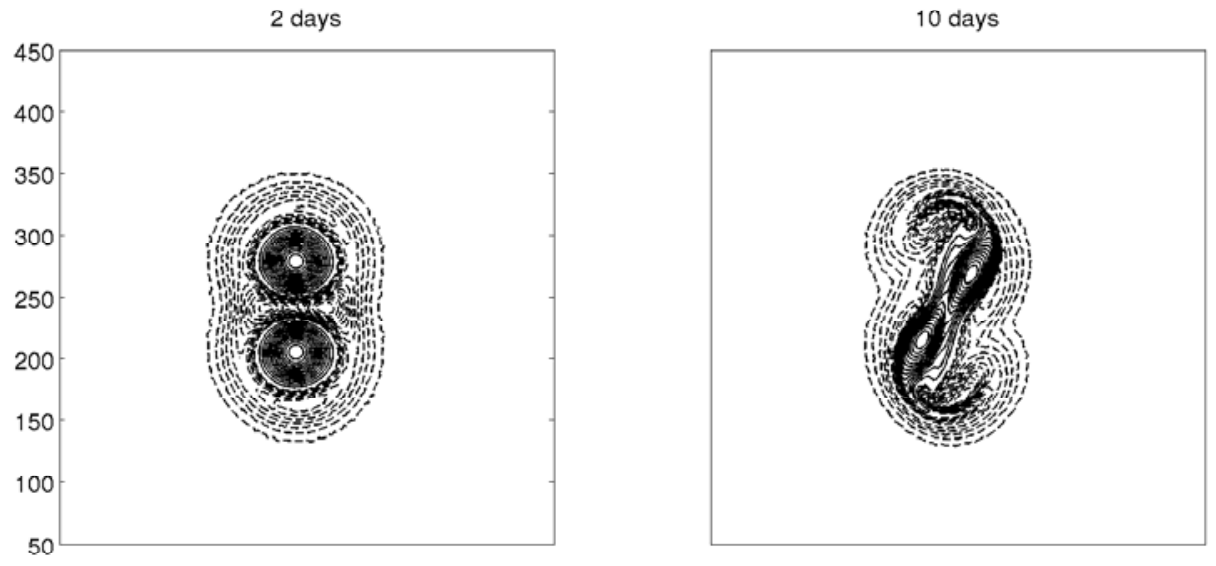

$y(\mathrm{~km})$
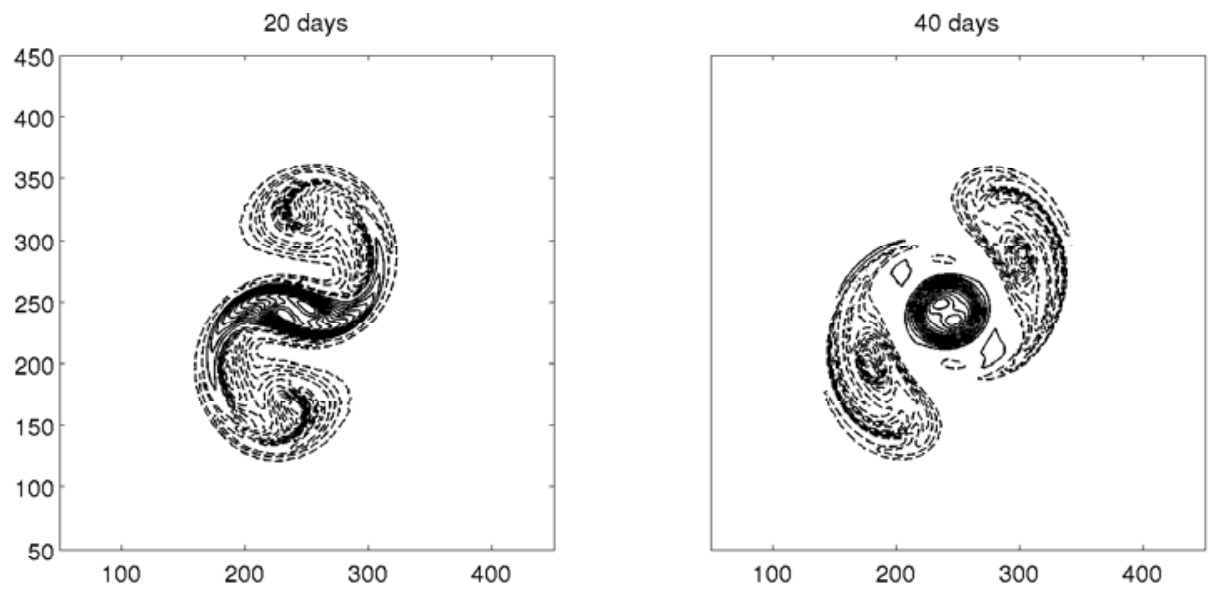

$x(\mathrm{~km})$

Figure 3. Horizontal sections of PVa at the vortex core. The relative initial distance between the subsurface anticyclones is $d_{c m}=2.5 R$ (M-vortices)

Table 2. Rossby numbers $(R o)$ and Stretching Factor $(S)$ for vortex merger experiments with changing rotational velocity EXP $1=$ slowest rotation, $, \ldots, \operatorname{EXP} 5=$ fastest rotation.

\begin{tabular}{cccccc} 
M-vortices & & & & & \\
\hline & EXP 1 & $\operatorname{EXP} 2$ & $\operatorname{EXP~} 3$ & $\operatorname{EXP} 4$ & $\operatorname{EXP~5~}$ \\
\hline$R o$ & 0.06 & 0.09 & 0.13 & 0.20 & 0.27 \\
$S$ & 0.24 & 0.32 & 0.35 & 0.44 & 0.50 \\
\hline
\end{tabular}

\begin{tabular}{ccccc} 
P-vortices & & & & \\
\hline & EXP 1 & EXP 2 & EXP 3 & EXP 4 \\
\hline$R o$ & 0.20 & 0.26 & 0.40 & 0.53 \\
$S$ & 0.35 & 0.41 & 0.92 & 0.92 \\
\hline
\end{tabular}



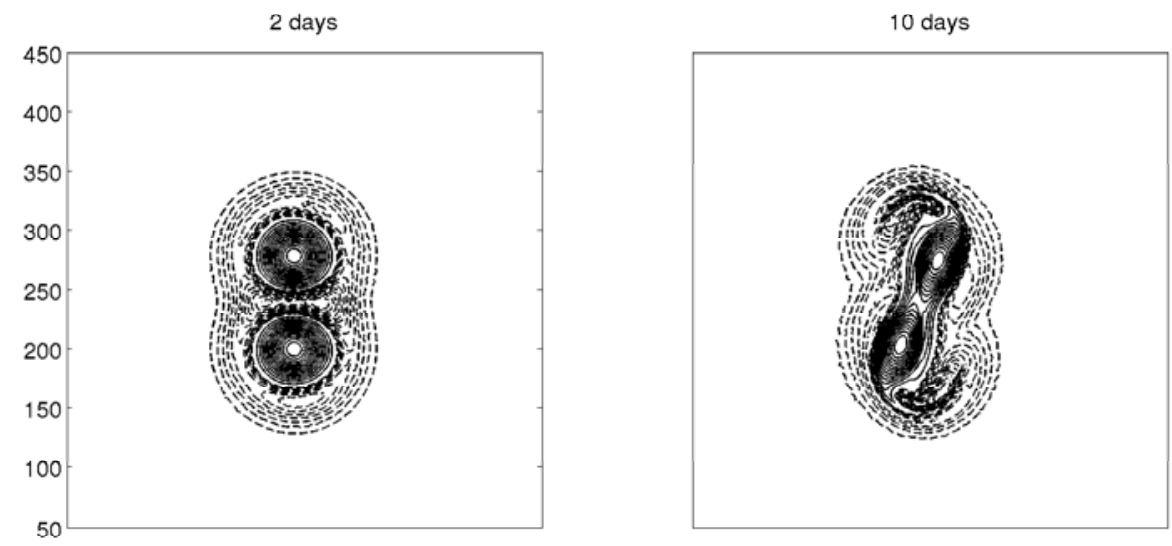

$y(\mathrm{~km})$
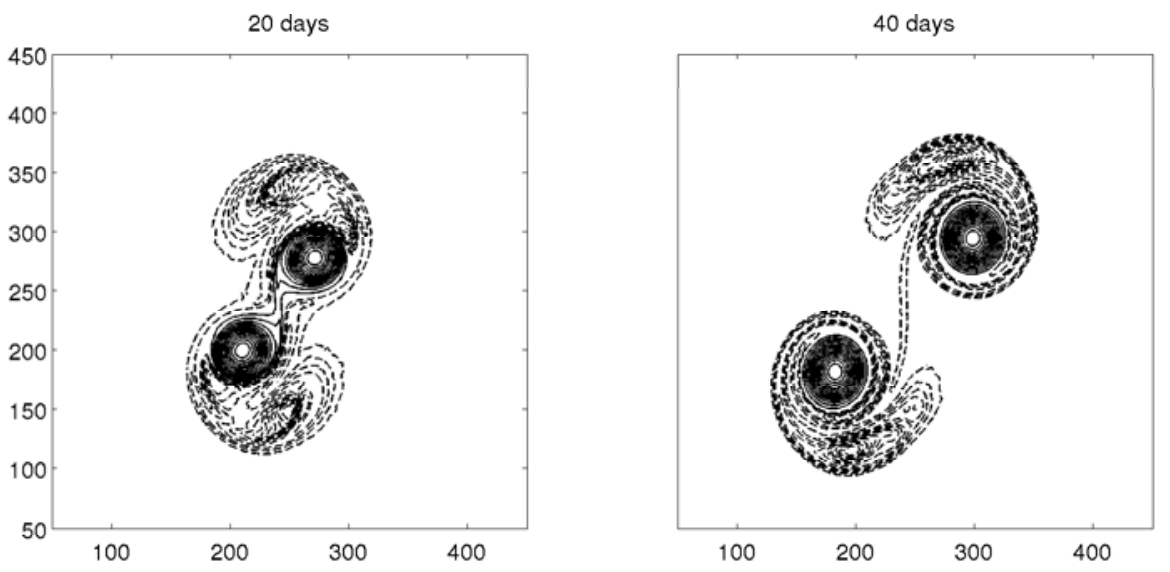

$x(\mathrm{~km})$

Figure 4. Horizontal sections of PVa at the vortex core. The relative initial distance between the subsurface anticyclones is $d=2.6 R$ (M-vortices)

If a critical initial distance is chosen, then the merger does not occur for rotational speeds lower than $0.15 \mathrm{~m} / \mathrm{s}$, for the $\mathrm{M}$ case. In this particular case, the two vortices interact for about one month and, afterwards, their cores separate giving rise to two coherent structures. This regime is then called Slight Interaction. On the other hand, for faster spinning velocities $(0.15 \mathrm{~m} / \mathrm{s}$ to $0.4 \mathrm{~m} / \mathrm{s})$, the merging process is achieved for progressively shorter times, inversely proportional to the vortex initial velocity. In the $\mathrm{P}$ case, the Slight Interaction regime is overcome at rotational speeds around $0.2 \mathrm{~m} / \mathrm{s}$, with a similar dependence of merger with respect to time. As for the $\mathrm{M}$ case, an increase in vortex rotational velocity results in an accelerated merging process. This is schematically summarized by figure 7 , where the light and dark grey areas indicate the slight interaction and merger regimes, respectively. In particular, the "NO MERGER" label stands for a regime in which the merger is possible but not achieved yet. 

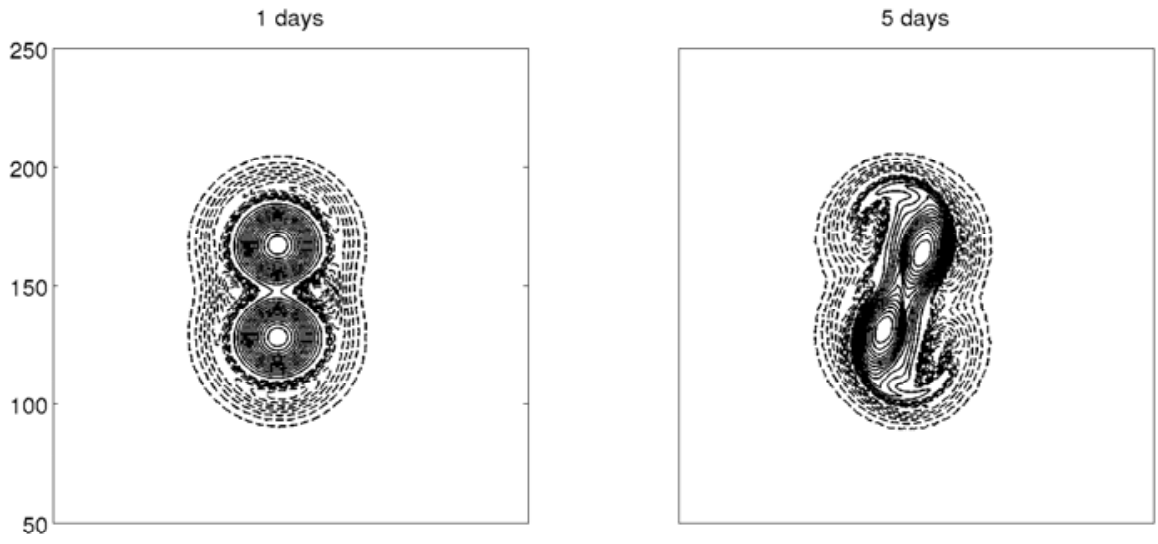

$y(\mathrm{~km})$
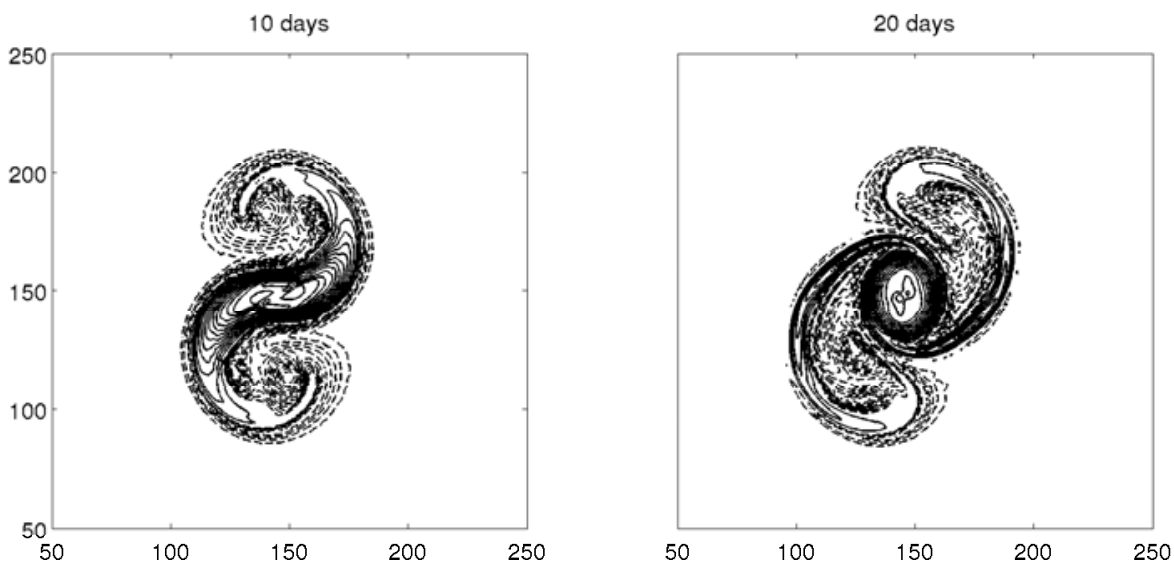

$x(\mathrm{~km})$

Figure 5. Horizontal sections of PVa at the vortex core. The relative initial distance between the subsurface anticyclones is $d=2.6 R$ (P-vortices)

\subsubsection{Influence of the background stratification}

Here are studied the effects of the background stratification on the merger of subsurface anticyclones. As we did for the case of initial rotational velocity, the study is carried out in a parametric way. We vary the background stratification in order to get associated Rossby deformation radii comprised between about 30 and $50 \mathrm{~km}$. In the real northern ocean, such values correspond to typical stratifications in the latitude range between $20^{\circ} \mathrm{N}$ and $30^{\circ} \mathrm{N}$ (Chelton et al. 1998), where subsurface anticyclones are abundant (Meschanov and Shapiro 1998 , Shapiro and Meschanov 1991, Pingree and Le Cann 1993, Armi and Zenk 1984, Richardson et al. 2000). In both the $\mathrm{M}$ and $\mathrm{P}$ case, we investigate the effects of stratification on vortices whose initial relative distance is the critical one, for instance, $d_{c M}=2.5 R$ and $d_{c P}=2.6 R$, respectively. The choice of the other initial parameters has already been discussed in section 2 and is schematically shown by figure 1. Note that the choice of $R_{d}$ range, of geographical interest, has also technical reasons. Considering the analytical structure of the subsurface an- 

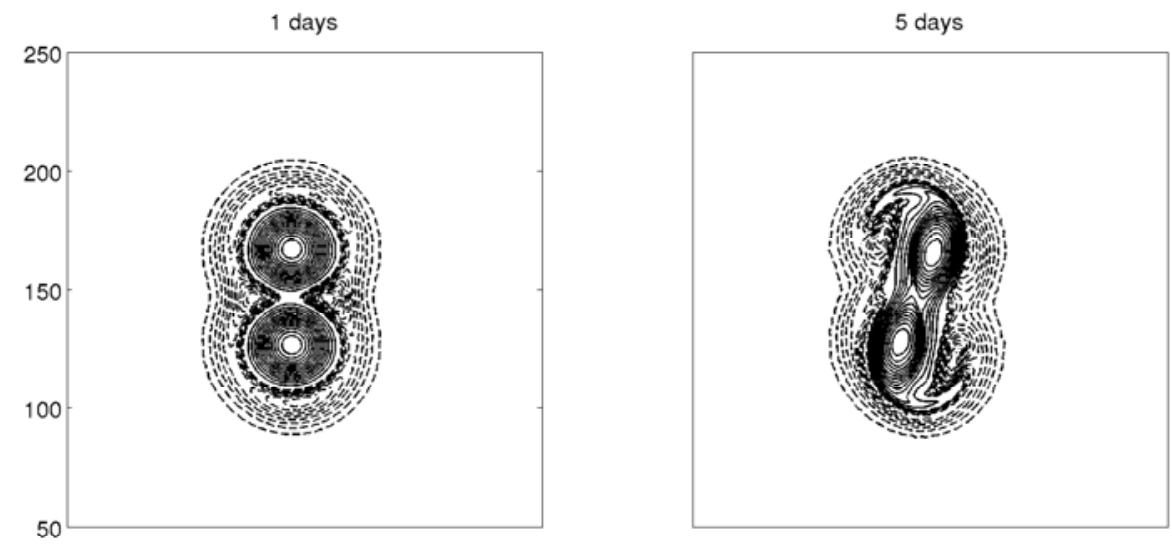

$y(\mathrm{~km})$
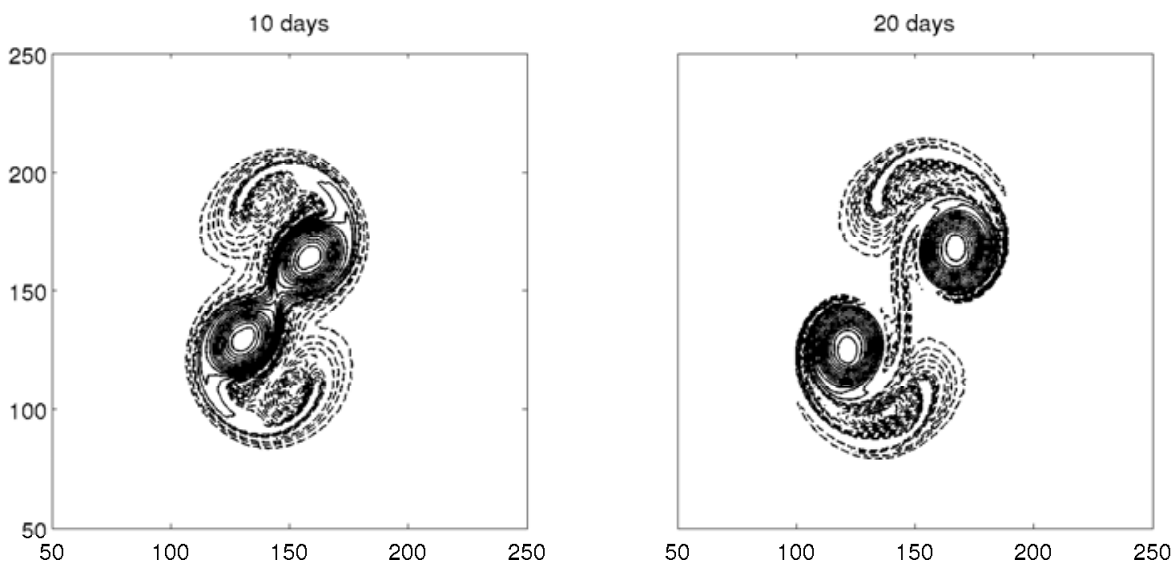

$x(\mathrm{~km})$

Figure 6. Horizontal sections of PVa at the vortex core. The relative initial distance between the subsurface anticyclones is $d=2.7 R$ (P-vortices)

ticyclones (specified in section 2), some values of $R_{d}$, for instance the smaller ones, can lead to statically unstable anticyclones, which are not of interest in this study.

The dimensionless numbers affected by this parametric study are the Burger number $(B u)$ and the Stretching Factor $(S)$. Their values for the corresponding experiments are listed in table 3. Here, the variation of $B u$ and $S$ is due to the varying background stratification. For instance, higher values of $R_{d}$ are obtained by choosing steeper background stratifications (i.e., higher values of $\left.\left|\partial_{z} \rho(x, y, z)\right|\right) . B u$ is a monotonic increasing function of $R_{d}$ (see also table 1) and its effects on the flow have already been discussed at the end of section 2. Moreover, as $R_{d}$ is incremented, the vortex core isopycnal layers will extend less in the vertical, as the vortex will be embedded in an increasingly stratified environment. Hence, larger values of $R_{d}$ yield smaller stretching factors.

In the $\mathrm{M}$ case, and in the observed stratification range, the merger time increases with increasing $R_{d}$. In particular, if $R_{d}$ varies from $30 \mathrm{~km}$ to approximately $50 \mathrm{~km}$, the time for subsurface anticyclones merger will increase from 1 month to 2 months. In the $\mathrm{P}$ case, the 
Table 3. Burger number $(B u)$ and Stretching factors $(S)$ for vortex merger experiments with changing stratification. EXP $1=$ lowest $R_{d}, \ldots$, EXP $4=$ highest $R_{d}$

\begin{tabular}{ccccc} 
M-vortices & \multicolumn{1}{l}{} \\
\hline & EXP 1 & EXP 2 & EXP 3 & EXP 4 \\
\hline$B u$ & 1.06 & 1.44 & 1.86 & 2.35 \\
$S$ & 0.36 & 0.35 & 0.26 & 0.19 \\
\hline
\end{tabular}

\begin{tabular}{ccccc} 
P-vortices & & & & \\
\hline & EXP 1 & EXP 2 & EXP 3 & EXP 4 \\
\hline$B u$ & 4.84 & 5.76 & 7.11 & 9.81 \\
$S$ & 0.89 & 0.58 & 0.56 & 0.48 \\
\hline
\end{tabular}

merger behavior is analogous to the $\mathrm{M}$ case. In fact, changing $R_{d}$ from $30 \mathrm{~km}$ to $50 \mathrm{~km}$, the time needed for merger is 2.7 times larger. Once again, this is schematically shown by figure 7 .

In past works, the background stratification was also found to influence the critical merging distance. Waugh (1992) showed that this quantity monotonically increases with $R_{d}$, while Filyushkin and Sokolovskiy (2011) found that it is a non-monotonic function of the Froude number (the Froude number being also a function of $R_{d}$ ). In our experiments, when $R_{d}$ is incremented, P-vortices merge at increasingly larger distances. If $R_{d}$ varies from $36 \mathrm{~km}$ to $52 \mathrm{~km}, d_{c P}$ goes from $2.6 R$ to $2.7 R$, and does not vary significantly for larger $R_{d}$. For Mvortices, considering a realistic range of $R_{d}$ values for typical Meddy latitudes $\left(20^{\circ} \mathrm{N}\right.$ to $\left.40^{\circ} \mathrm{N}\right)$, no substantial variations of the critical merging distance were evidenced.

\subsubsection{Further considerations on the dimensionless hydrodynamical numbers}

Here we discuss the influence of the hydrodynamical numbers (listed in tables 2 and 3 ) on the vortex merger. These numbers characterize the hydrodynamical regime in which the merger experiments take place. At the end of section 2, the hydrodynamical context was discussed for $\mathrm{M}$ and P-vortices in their "reference-shape", i.e., with characteristics typical of Meddies and Peddies observed in the ocean. Here we study the impact of varying the vortex swirl velocity and environmental stratification on the merger.

When the vortex swirl velocity increases from $0.15 \mathrm{~m} / \mathrm{s}$ to $0.4 \mathrm{~m} / \mathrm{s}$, and the vortex radius is kept constant, the Rossby number monotonically increases (as shown in table 2). In our case $R o$ reaches its maximum value $(R o=0.53)$ for P-vortices spinning at $0.4 \mathrm{~m} / \mathrm{s}$.

When $R o$ approaches unity, the flow switches from geostrophic to unbalanced dynamics, and in the limit of $R o=1$ anticyclonic eddies can undergo centrifugal instability (see also Molemaker et al. 2014). In our study, even the less geostrophic anticyclones (i.e., $R o=0.53$ ) are far from this threshold. These conditions assure that the anticyclonic eddies we deal with, are keen to maintain their coherent structure.

Furthermore, increasing $R o$ will affect merger. If the critical conditions for merger are satisfied, the shear of vortex swirl velocity determines the rate at which the two vortex cores will exchange water masses. Hence, larger swirl velocities will accelerate the merging process. This is confirmed by comparing the stream function of two couples of merging M-vortices spinning at $0.15 \mathrm{~m} / \mathrm{s}$ and $0.4 \mathrm{~m} / \mathrm{s}$, respectively (evaluated at $1000 \mathrm{~m}$ depth and in the rotat- 

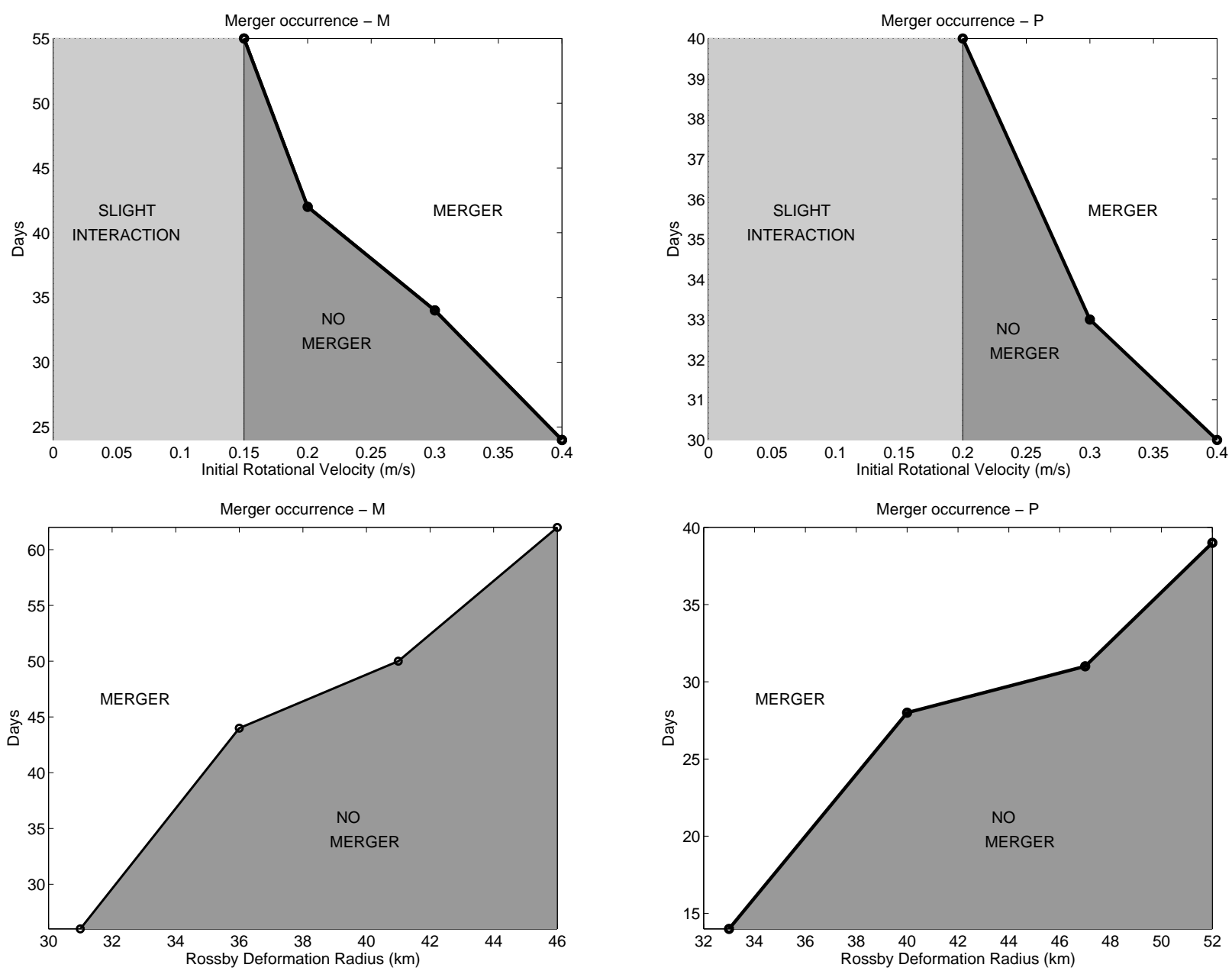

Figure 7. Time required for subsurface vortex merger. Top-left and top-right images show the dependence on initial rotational velocity in a $\mathrm{M}$ and $\mathrm{P}$ case, respectively. Analogously, bottom-left and bottom-right images show the dependence on the Rossby deformation radius. In the figure, the dots correspond to the experiments actually run in our study.

ing framework of the two co-rotating vortices). In the second case, larger stream function and velocity gradients bound the area around the centers of the two initial anticyclones; i.e., the area involved in the merging process (not shown). The larger shear of the vortex velocity field (compared to the $0.15 \mathrm{~m} / \mathrm{s}$ case) will favor destabilization and will lead to a more efficient water exchange between the cores of the anticyclones.

As stated in the previous section, when one increases the vortex swirl velocity without changing the vortex radius, the subsurface anticyclones are characterized by an increasing Stretching factor $S$, indicating that the isopycnal layers associated to the anticyclones core will be more stretched in the vertical.

In table 3 , the tendencies of both the Burger number $B u$ (increasing) and the Stretching factor $S$ (decreasing), indicate that when $R_{d}$ is increased, the motion progressively switches to a two-dimensional fashion.

Except for M-vortices in their "reference shape", where $B u$ is of order unity, all the other configurations indicate a predominance of relative vorticity over stretching. $B u$ reaches its maximum value (around 10) for P-vortices embedded in the most stratified environment, i.e., when $R_{d} \simeq 52 \mathrm{~km}$ (Cushman-Roisin and Beckers 2011). In addition, the vertical structure 
of the vortex cores will extend less in the vertical as $R_{d}$ is incremented (as expressed by the decaying $S$ values).

In our experiments, the increase in $R_{d}$ also resulted in a progressively larger merging-time for both the couples of $\mathrm{M}$ and $\mathrm{P}$-vortices. This behavior can be explained observing equation 7 . When $R_{d}$ is incremented, the quantity $\left|\partial_{z} \rho(x, y, z)\right|$ becomes larger. Hence, the PVa gradients across the two vortex cores will increase. As a result, merger will need more time to balance such gradients and eventually yield the single contour core (which is the condition that we defined as merging-time).

\section{Merger related effects}

\subsection{Evolution of PVa volume integral}

Here we quantify how merger modifies the structure of subsurface anticyclones. In particular, we determine the vortex evolution as a function of time, making use of its three-dimensional PVa integral. This quantity is directly related to the vortex stream function, hence, to its velocity field. A simple way to look at this problem is to consider three-dimensional quasigeostrophic vortices (Carton 2001). For idealized oceanic vortices, the stream function of a vortex is the convolution of the PVa with the Green's function. Hence, higher PVa volume integrals will yield faster rotating vortices. The PVa analysis will be carried out for both $\mathrm{M}$ and P-Vortices. At first, we determine the time evolution of the PVa volume integral in a case of merger and in another in which vortices only slightly interact. The merger case results from an initial state in which two subsurface anticyclones (as the ones illustrated in figure 1) are separated by distances $d_{c M}$ and $d_{c P}$ and spin at approximately $0.2 \mathrm{~m} / \mathrm{s}$. The slight interaction is instead obtained increasing $d_{C M}$ and $d_{c P}$ by $0.1 R$. Four experiments are then performed. The set of dimensionless numbers for the $\mathrm{M}$ and $\mathrm{P}$ cases are given in table 4 . The dimensionless hydrodynamical numbers have already been discussed in sections 2 and 3 .

Table 4. Characteristics of the modelled subsurface vortices for determination of the PVa volume integral.

\begin{tabular}{ccccccc}
\hline & $B u$ & $R e$ & $R o$ & $S$ & $d_{c M E R G}$ & $d_{c S L I C H T L N T}$ \\
\hline M-vortices & 1.36 & $\gg 1$ & 0.13 & 0.35 & $2.5 R$ & $2.6 R$ \\
P-vortices & 5.42 & $\gg 1$ & 0.26 & 0.41 & $2.6 R$ & $2.7 R$ \\
\hline
\end{tabular}

In both the merger and slight interaction cases, the PVa volume integral has been evaluated taking into account the contribution of the negative PVa core only. This is justified by the fact that the annuli, as well as the positive lobes, are not coherent structures throughout the merging (or slight-interaction) process, but they undergo strong deformation and filamentation (see for example figure 13).

In particular, in the slight interaction case, the contribution of a single vortex is considered to establish PVa budgets. Given the symmetry of our configuration, the "southern-vortex" has been arbitrarily selected, putting to zero all the PVa values located northeast of the separation line (dash-dotted line in figure 8). Its displacement has been automatically tracked in the oceanic basin, and the PVa integral has been evaluated in a moving cylinder, centered 

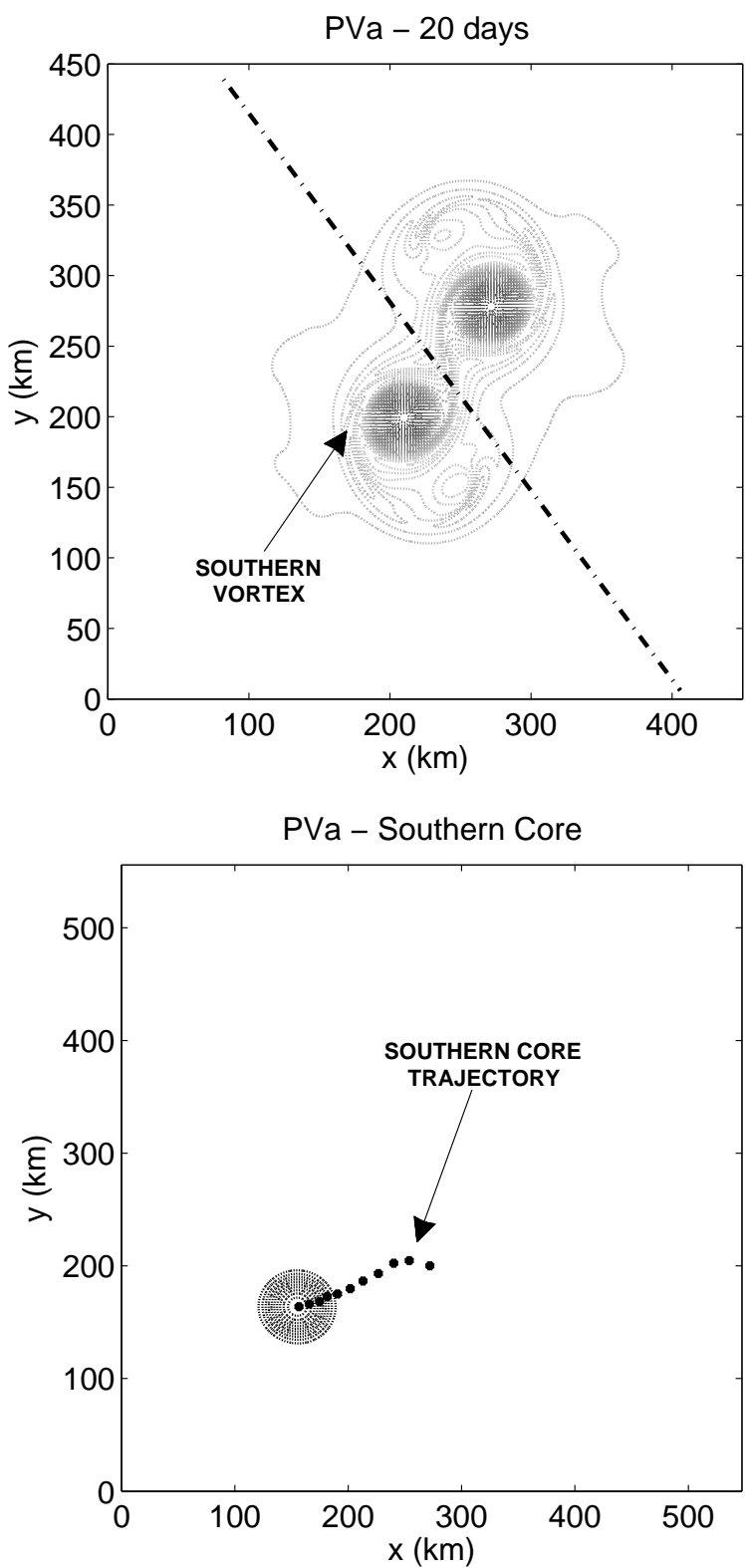

Figure 8. Tracking of a single subsurface vortex for evaluation of the PVa volume integral in the slight interaction case. Top: separation line between the two vortices (dash-dotted line), Bottom: trajectory of the PVa negative core.

at the vortex core and whose radii are around $60 \mathrm{~km}$ and $40 \mathrm{~km}$ in the $\mathrm{M}$ and $\mathrm{P}$ case, respectively. The evaluation of the PVa volume integral becomes much easier when merger occurs, as the vortex becomes nearly steady after about 20 days, making its tracking a trivial task. The cylinder in which the integral is evaluated has the same characteristics as in the slight-interaction case.

Performing the computation in such cylinders, allows one to evaluate the PVa integral on the main coherent structure of the vortex, neglecting filaments and production of smaller eddies.

The results for $\mathrm{M}$ and $\mathrm{P}$ cases are shown in figure 9. When vortices slightly interact, the core PVa integral is fairly constant throughout the simulation.

On the other hand, when merger occurs, the two vortex cores have to be taken into account 


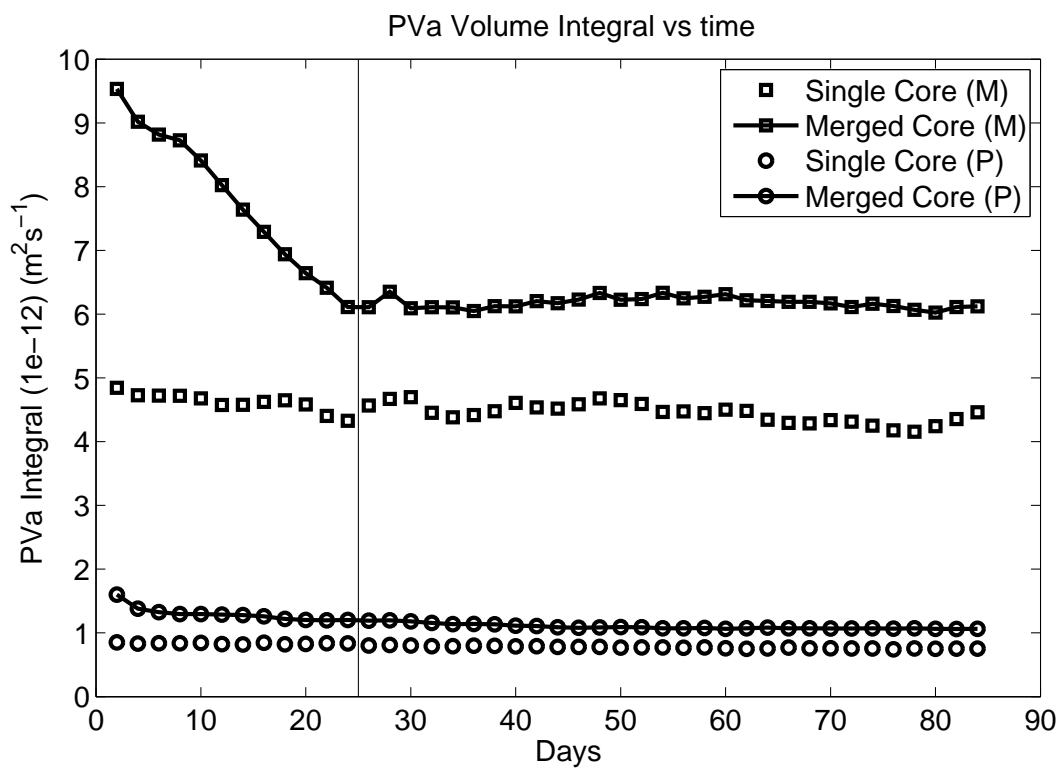

Figure 9. PVa integral for the core of the subsurface vortex. Squares and circles refer to the PVa integral for M and P-vortices, respectively, with unfilled symbols corresponding to the "Slight Interaction" case (single vortex) and filled symbols corresponding to the "Merger" case (two vortices). The thin vertical line indicates the time when a single coherent structure is generated by the merger of the two initial vortices.

and the PVa integral is initially doubled compared to the slight interaction case. As the system evolves, the PVa integral monotonically decreases until merger is completed (around day 25, vertical line of figure 9). Afterwards, the PVa integral of the merged core becomes constant with time; yet its value is larger than in the slight interaction case. The monotonic decrease of PVa integral (for merging vortices) indicates that part of the total PVa, initially stored in the two vortex cores, is lost due to filaments (in addition to the filamentation of the PVa annuli).

The filamentation processes occurring during merger, associated to a migration of PVa from the core of the vortex towards its periphery, can be quantified by means of the Merger Efficiency (ME hereinafter), similarly to Waugh (1992). In our case, this quantity is expressed by the ratio of PVa integral at the end of the simulation (around day 80) over its value computed at time $t=0$, as shown by (9),

$$
M E=\frac{\int_{V} P V a_{f}(x, y, z) \mathrm{d} V}{\int_{V} P V a_{i}(x, y, z) \mathrm{d} V},
$$

where $V$ indicates the volume of integration (the cylinder) and the subscript $i$ and $f$ stand for initial and final state of the simulation, respectively. The computed ME values are 0.64 and 0.67 in the $\mathrm{M}$ and P-case, respectively. Earlier works on the merger of two-dimensional Rankine and Gaussian vortices suggest that the ME can be higher than the one obtained in this context, with values generally exceeding 0.8 (Waugh 1992, Trieling et al. 2005). Our experiments indicate that for both $\mathrm{M}$ and $\mathrm{P}$-vortices, the merger induces a non negligible filamentation, causing a partial loss (around 35\%) of the PVa associated to the vortex cores. This different behavior is due to the unlike-signed PVa of the three-dimensional anticyclones we dealt with. During merger, the filamentation of the vortex cores (as shown by the continuous contours in figure 3,5 and 13 is particularly enhanced across the areas where the relative vorticity (hence the PVa) changes in sign, i.e., at the cores peripheries. In this regions, the strain increases and 
reaches its maximum values, favoring the vortex deformation with the consequent migration of water masses away from the centers of the vortices (not shown).

Nevertheless, the merging process yields a single vortex whose PVa integral is larger than that of the initial vortex core. Indeed, averaging the values of the PVa integral from day 25 onward, we can estimate that the PVa gain due to merger is around $39 \%$ and $42 \%$ in the M and $\mathrm{P}$ case, respectively. This has been computed using (10):

$$
P V a_{\mathrm{GAIN}}=\frac{\left\langle\int_{V} P V a_{\mathrm{Merge}}(t>25) \mathrm{d} V\right\rangle_{[\mathrm{M} / \mathrm{P}]}}{\left\langle\int_{V} P V a_{\text {Single Core }}(t>25) \mathrm{d} V\right\rangle_{[M / P]}}-1,
$$

where $t$ is the time in days and the subscript $[\mathrm{M} / \mathrm{P}]$ indicates $\mathrm{M}$ and $\mathrm{P}$-vortices, respectively.

\subsection{Vortex deformation}

Another merger-induced effect, which was evident in all the simulations, is the deformation of the two vortices before and after the merging process has taken place.

The subsurface anticyclones initially have a circular shape. If their relative distance is the critical one, they start exchanging potential vorticity, and exhibit an elliptic shape. This deformation is evident up to the latest stages of the simulations, as shown by Figures 10 and 14. In order to quantify this phenomenon, we compute the geometrical moment for one of the two subsurface vortices before the merging process and on the merged structure afterwards; for both $\mathrm{M}$ and $\mathrm{P}$ vortices. The geometrical moments, expressed by (11), allow us to define which are the preferential deformation modes (see (12)) of our three-dimensional structure all along the simulation. In particular, in (11), the following quantities have been introduced:

- $\left(x_{c}, y_{c}, z_{c}\right)$ the coordinates of the vortex center in the oceanic basin;

- $R$ the vortex radius;

- $H$ the vortex thickness;

- pva, the normalized PVa:

$$
\begin{gathered}
M_{m n p}(t)=\iiint\left(\frac{x-x_{c}}{R}\right)^{n}\left(\frac{y-y_{c}}{R}\right)^{m}\left(\frac{z-z_{c}}{H}\right)^{p} \operatorname{pva}(x, y, z, t) \mathrm{d} x \mathrm{~d} y \mathrm{~d} z \\
\operatorname{Mode}_{i}(t)=\sqrt{\left(M_{i 00}\right)^{2}+\left(M_{0 i 0}\right)^{2}+\left(M_{00 i}\right)^{2}}
\end{gathered}
$$

Before the merging process, the computation of the deformation modes is performed isolating the core of one of the two subsurface anticyclones (the southern one, as explained in section 4.1). The isolation consists in neglecting one of the two vortices and its PVa shields, as schematically shown in figure 10. After the merger, the only required operation is the elimination of the PVa shield. Note that in this particular analysis, we define the "after-merger" by visual inspection, observing when a single coherent core is formed.

The results of this analysis are shown in figure 11. In both the $\mathrm{M}$ and $\mathrm{P}$ case, a clear dominance of the mode 2 is evident all along the simulation, confirming our prediction. Note that, in the initial stages, mode 3 grows as the vortices come close to each other. This mode corresponds to the "pear-shaped" equilibria for two close two-dimensional vortices Overman 
et al. 1981 .
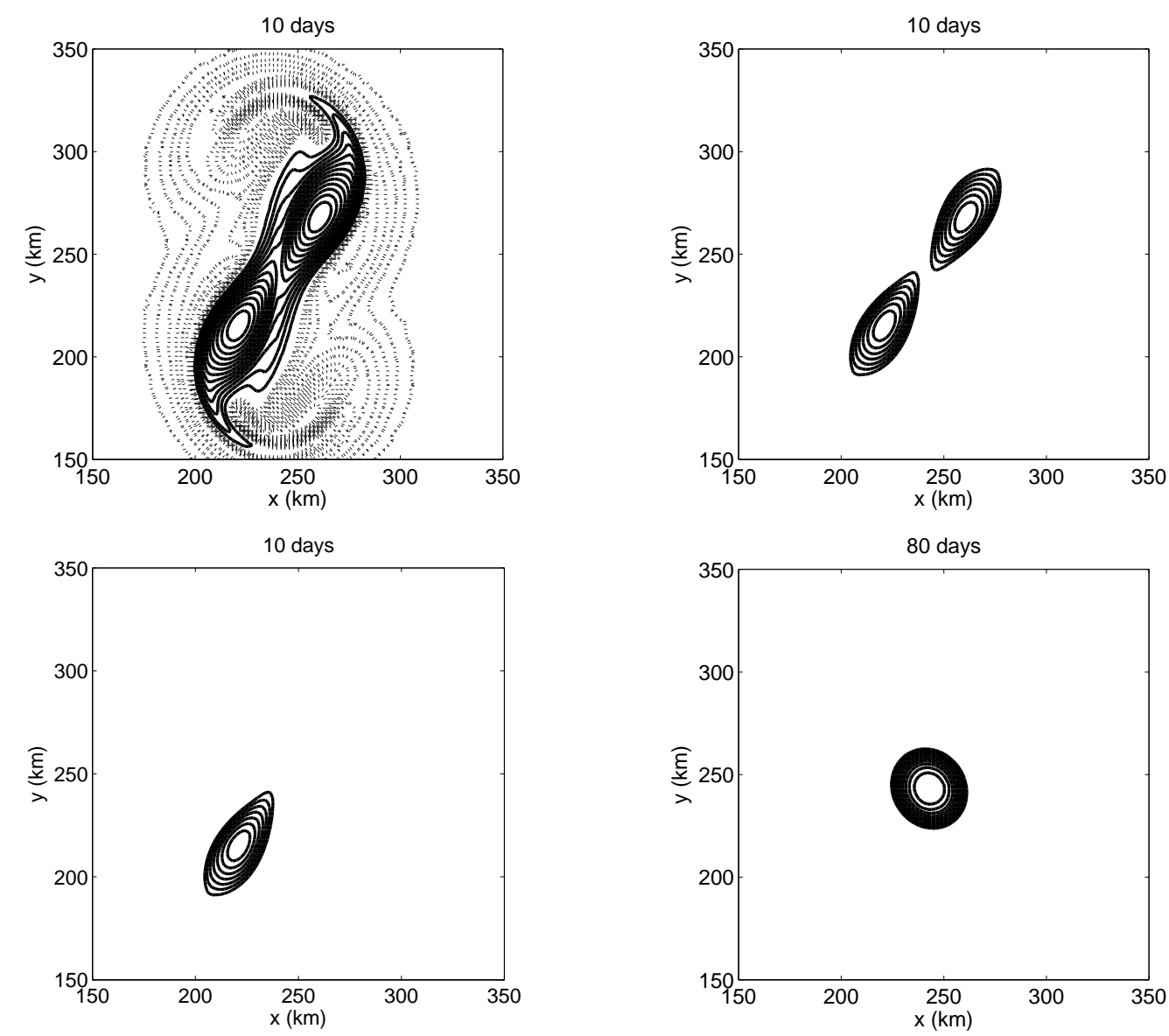

Figure 10. Isolation of a single subsurface vortex for evaluation of the geometric moment. The images show a snapshot of PVa for two M anticyclones during merger (10 days) and after merger (80 days)

\subsection{Final PVa structure of the merged vortex}

One remarkable feature is the PVa structure after the merging process has taken place. For both $\mathrm{M}$ and $\mathrm{P}$-vortices, horizontal sections of $\mathrm{PVa}$ indicate the generation of multipoles. Visual inspection suggests that this feature becomes evident after approximately 50 days in the $\mathrm{P}$ case and 100 days in the $M$ case (note that in this section simulations have run for 200 days at a horizontal resolution of $500 \mathrm{~m}$ ). The main PVa core is surrounded by two satellite small vortices (labelled as E1 and E2) whose radii can be as small as $10 \mathrm{~km}$ (see figure 14) and are generated by the instability of peripheral flaments (as shomn by figure 10 for M-vortices; not shown for P-vortices). Vertical profiles of kinetic energy evaluated through the center of these eddies indicate that their vertical extension can reach several hundred meters (figure 12). In the end, the lifetime of these small-scale features can reach or exceed five months, as indicated by the latest stage of the simulations.

The vertical section of PVa shows that the merged vortex maintains some of the features of the initial state. As stated in section 2, the initial PVa structure couples the effect of both R-vortices and S-Vortices (Morel and McWilliams 1997), and this is still the case for the final 

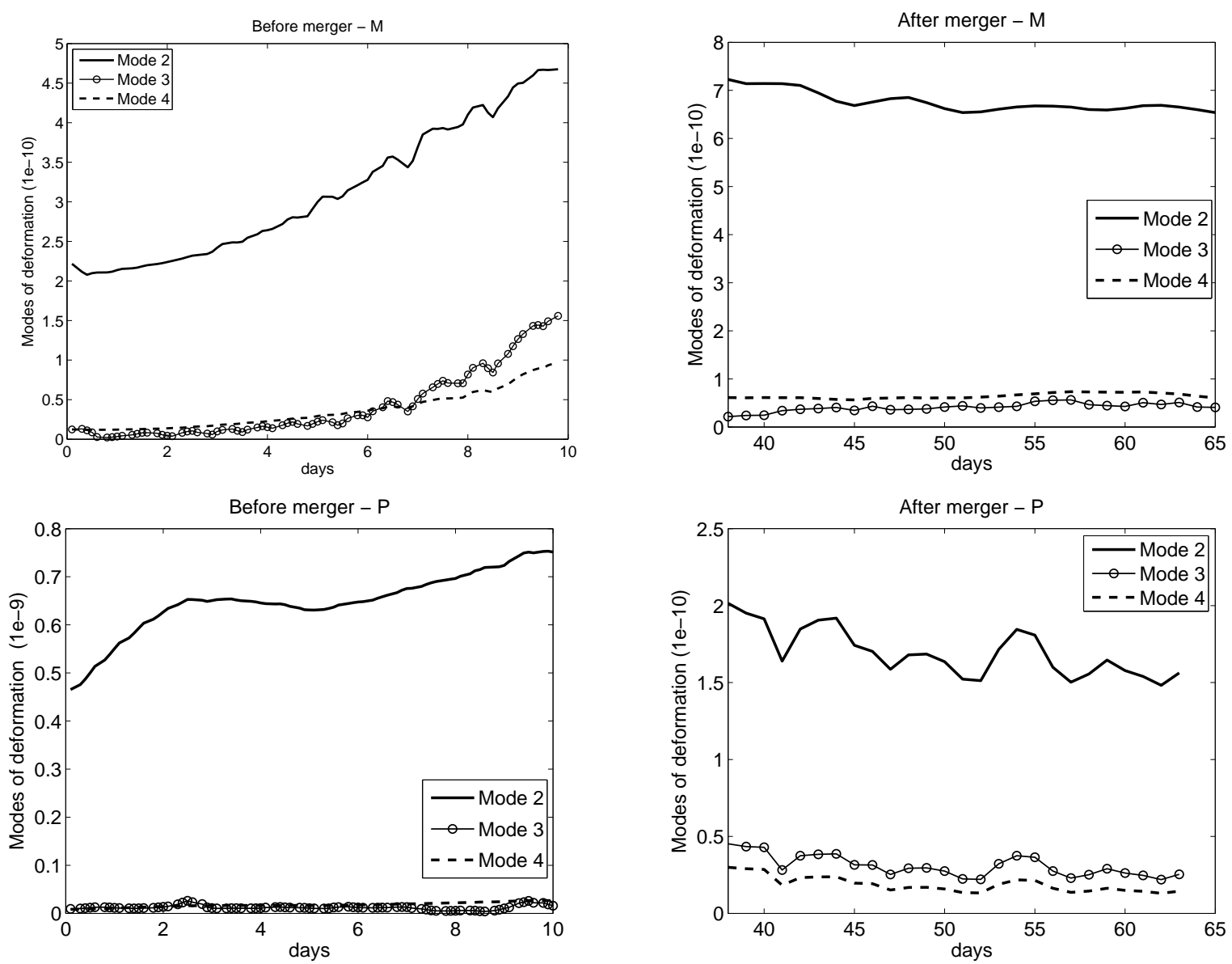

Figure 11. Deformation modes for the subsurface vortex merger. Top-left and top-right figures are related to M-vortices, before and after the merger, respectively. Results for P-vortices are shown in the bottom-left and bottom-right figures.

vortex. In fact, patches of opposite sign PVa still surround and vertically bound the merged vortex. However, the horizontal structure exhibits a tripole-like configuration, though many small vortices and filaments orbit the main vortex core. Vertically, the PVa shield is fragmented into upper and lower lobes. The merged structure has radii and thicknesses comparable to those of the initial vortices. In the $\mathrm{M}$ case, the merged vortex increases its radius by about $30 \%$ and decreases its thickness by $20 \%$, while, in the $\mathrm{P}$ case, the percentages are around $18 \%$ and $9 \%$, respectively. These values have been estimated focusing on the variations of the negative core, neglecting the noisier area given by the positive PVa shields.

These results are also in agreement with the work of Bashmachnikov et al. (2015). The authors, using in-situ data, found that Meddies progressively increase in radius as they drift west of the Iberian Peninsula; within distances of $600 \mathrm{~km}$ from the Portuguese coasts. They pointed out that merger is the main mechanism responsible for this behavior. Moreover, they observed that Meddy thicknesses only slightly decrease as merger events take place. Hence, in the real ocean, the Meddy aspect ratio is a decreasing function of the distance from the Iberian peninsula, i.e., as merging events are produced, as also shown by our idealized experiments.

In summary, the set of dimensionless numbers describing the effects of merger on subsurface anticyclones are illustrated in table 5. The Rossby and Burger numbers will slightly decrease because of the increasing radii. Moreover, the merging process does not significantly change 

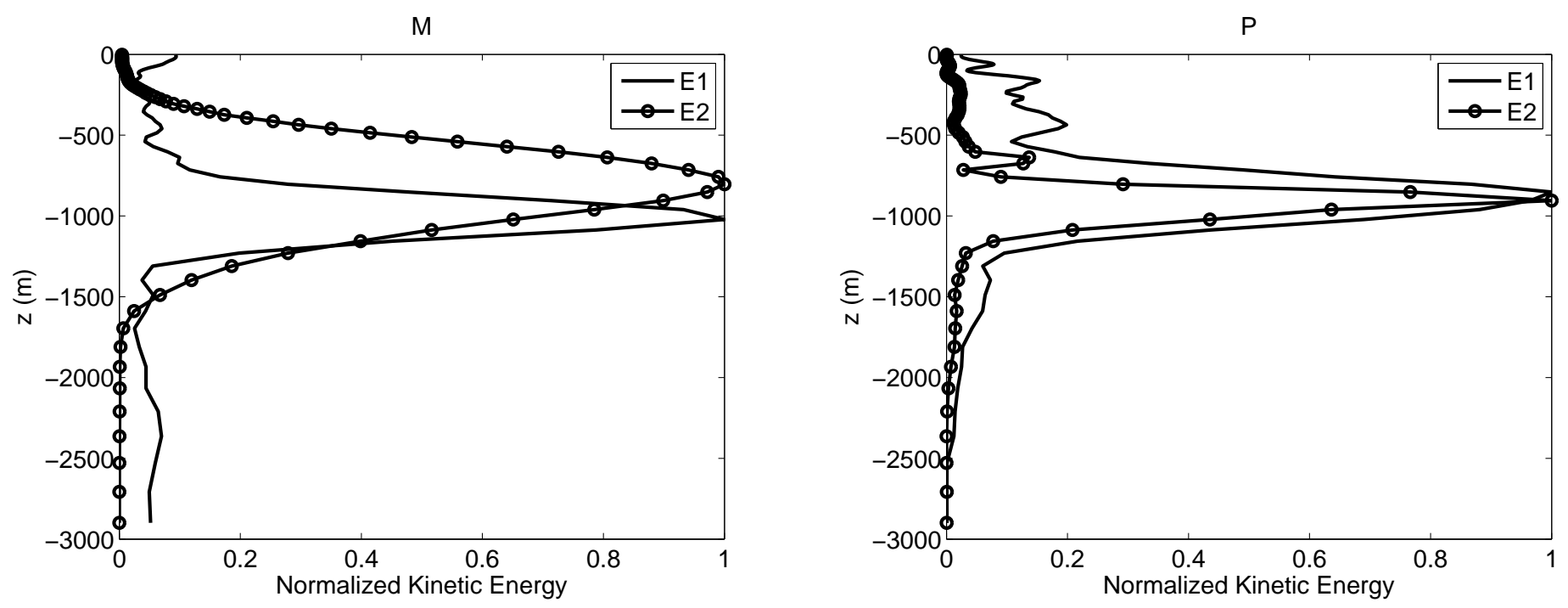

Figure 12. Vertical profiles of kinetic energy through the centers of the eddies E1 and E2 (see also figure 14). Results are presented for $\mathrm{M}$ and P-Vortices.
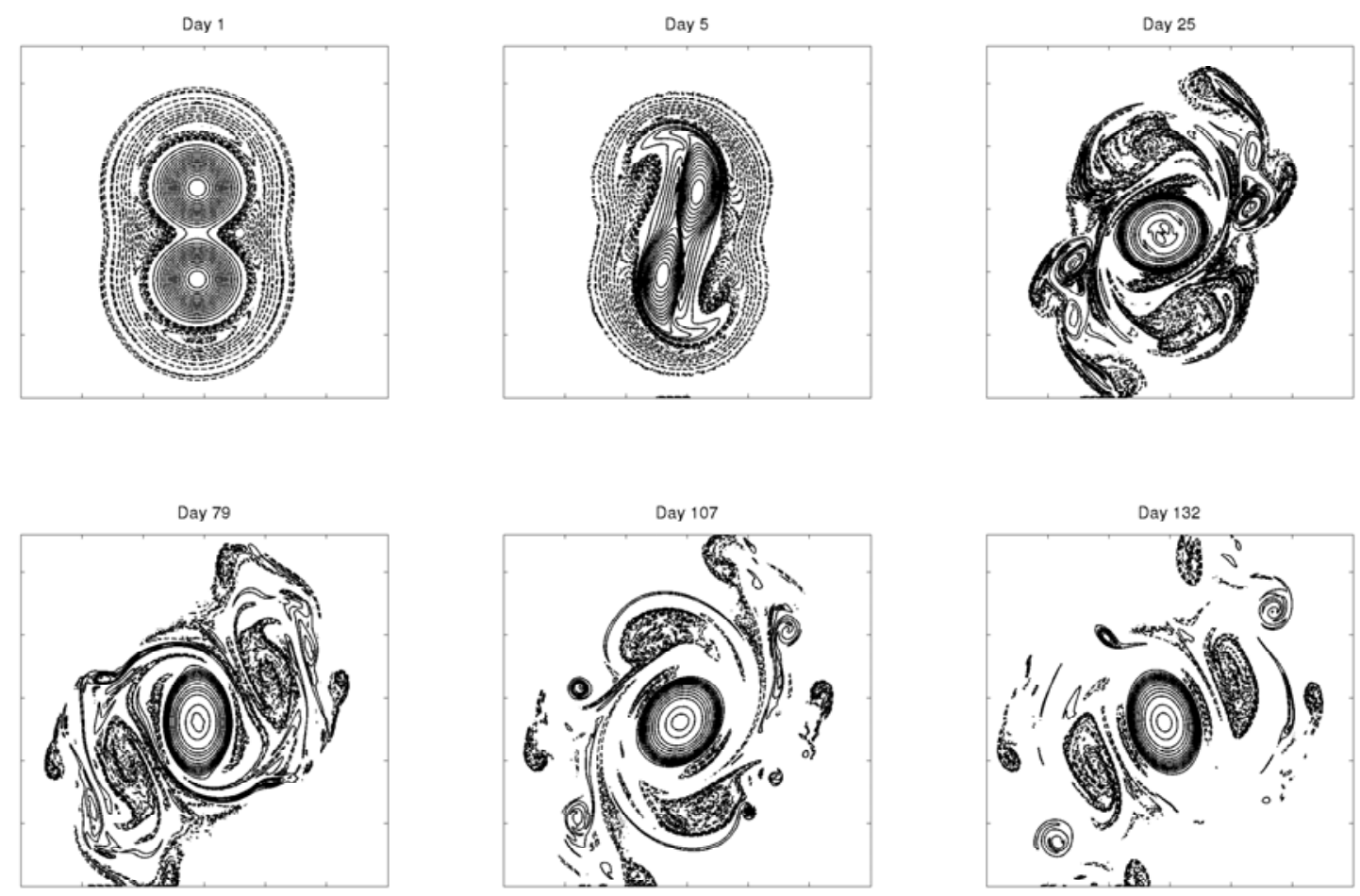

Figure 13. Generation of peripheral eddies via filament instability (M-vortices).

the isopycnal structure of the vortex. The stretching factors are in fact comparable before and after the merger.

The implications of the vortex characteristics on merger have already been discussed in the previous sections. Nevertheless, the different Burger numbers characterizing the $M$ and 

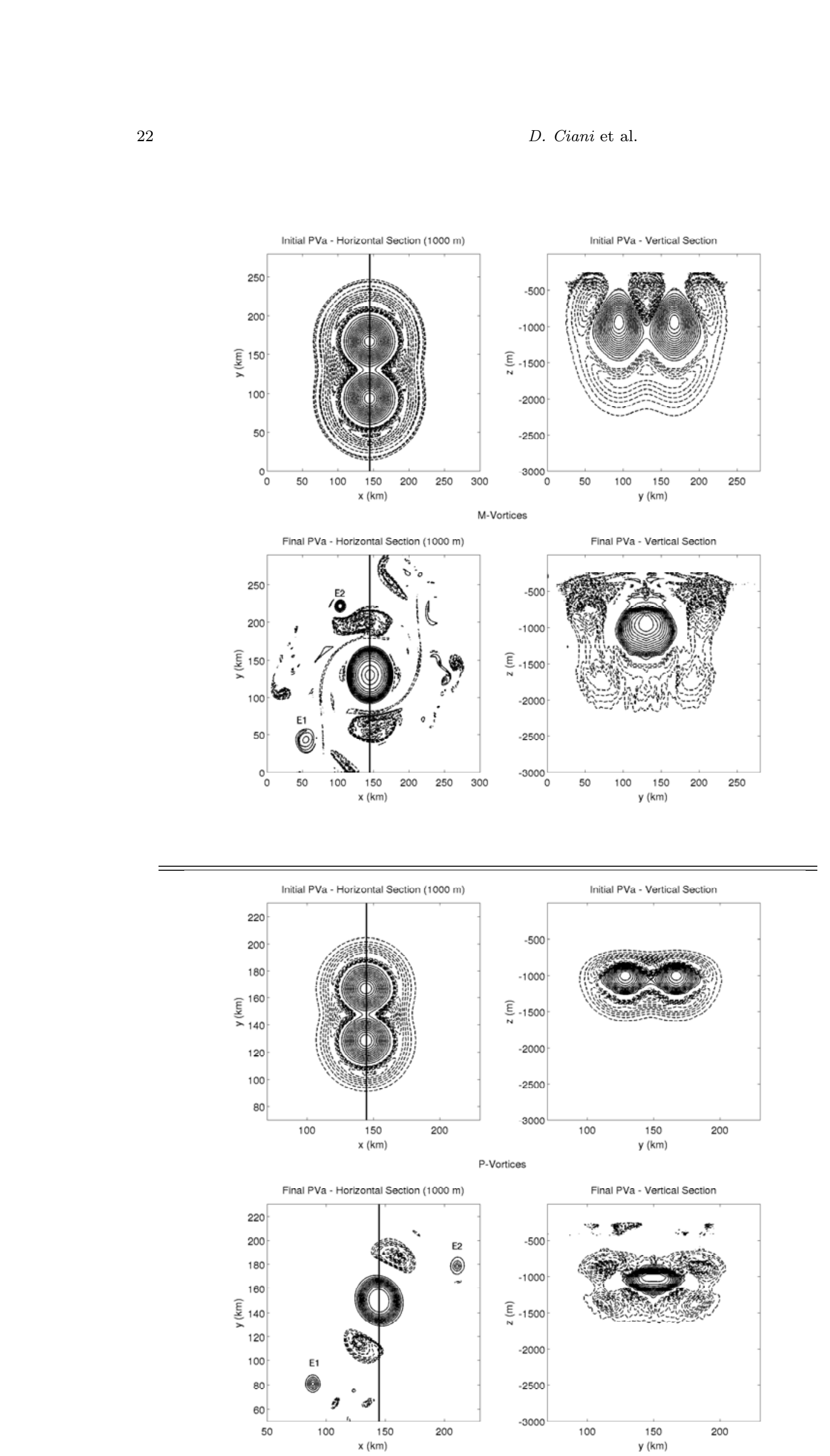

Figure 14. Initial and final sections of PVa for merger of subsurface anticyclones (M and P-Vortices). The vertical sections are estimated along the radial indicated by the bold vertical line. The peripheral eddies are labelled as E1 and

E2 in both cases.

M-Vortices$$
\text { E2 in both cases. }
$$ 
P-vortices $\left(B u_{M}=0.9, B u_{P}=4.25\right)$ are worth commenting. As pointed out at the end of section 2, when $B u>1$ the flow is predominantly two-dimensional (Cushman-Roisin and Beckers 2011), as relative vorticity mostly drives the motion. On the other hand, a Burger number of order unity indicates a regime in which rotation and stratification equally contribute to determine the flow; giving the PVa-distribution a more three-dimensional character. Indeed, maps of vertical velocity evaluated at the final stage of the simulations in correspondence of the vortex core (not shown) confirm this behavior. In the M case, upward velocities at $1000 \mathrm{~m}$ depth are larger than in the $\mathrm{P}$ case, as we observe upward motions up to $4 \mathrm{~m}$ /day against the value of $2 \mathrm{~m} /$ day observed for P-vortices. Moreover, such vertical displacements are spatially correlated with the main filaments located around the merged core (see lower-left panel of figure 14 for M-Vortices); and filaments are known to be regions of enhanced vertical motions (Ponte et al.|2013). However, such vertical velocities still allow the motion to be predominantly two-dimensional, as the core of the merged M-vortex swirls at a maximum speed of $0.28 \mathrm{~m} / \mathrm{s}$.

Table 5. Hydrodynamical numbers for the modelled subsurface vortices before and after the merger.

\begin{tabular}{ccccc} 
M-vortices & \multicolumn{1}{c}{ Ro } & $S$ \\
\hline BEFORE Merger & 1.36 & $\gg 1$ & 0.13 & 0.36 \\
AFTER Merger & 0.90 & $\gg 1$ & 0.10 & 0.40 \\
\hline
\end{tabular}

\begin{tabular}{ccccc}
\multicolumn{1}{c}{ P-vortices } & $B u$ & $R e$ & $R o$ & $S$ \\
\hline BEFORE Merger & 5.42 & $\gg 1$ & 0.26 & 0.41 \\
AFTER Merger & 4.25 & $\gg 1$ & 0.20 & 0.37 \\
\hline
\end{tabular}

\section{Conclusions}

In this paper we have investigated the merger of subsurface lens-like anticyclones in the context of the full primitive equations. The study has been carried out by means of the Regional Oceanic Modeling System (ROMS) in academic configuration and neglecting any atmospheric forcing or interaction with topographic features. This choice is justified by the evidence of colliding lens-like isolated anticyclones off of Gibraltar Strait (referred to as Meddies) confirmed by past and recent studies based on observations and realistic modeling (Schultz Tokos et al. 1994, Barbosa Aguiar et al. 2013, L'Hégaret et al. 2014). Furthermore, this study also provides an idealized description for the merger of smaller subsurface eddies, as the ones recently observed in the north-western Indian Ocean (Peddies) by means of in-situ measurements (L'Hégaret 2015). Meddies and Peddies (respectively, Mediterranean and Persian-water eddies) are both subsurface eddies generated by the sinking of salty and warm water masses in semi-closed basins and their subsequent outflow into the open ocean. The first are characterized by radii comprised between 20 and $50 \mathrm{~km}$ and they can extend vertically from some hundred meters up to $1 \mathrm{~km}$ while the second have smaller radii and vertical extension. Both 
of such structures spin at velocities around $0.2 \mathrm{~m} / \mathrm{s}$. In our study, these oceanic eddies are studied by means of analytical three-dimensional fields, i.e., the density and the velocity field.

The critical parameters for merger occurrence have been determined. If a reference state is chosen, in which two subsurface anticyclones spin at about $0.2 \mathrm{~m} / \mathrm{s}$, the critical merger distances are $d_{c M}=2.5 R$ and $d_{c P}=2.6 R$ for $\mathrm{M}$ and P-Vortices, respectively (with $R$ the vortex radius and $\mathrm{M} / \mathrm{P}$ indicating Meddy-like and Peddy-like lenses). This parameter can also be affected by background oceanic stratification and vortex rotational velocity. In particular, we found that faster rotating lenses living in less stratified environments, provided the conditions for merger, require smaller times to complete the merging process. Slower rotating lenses merge with increasing times and may need smaller critical distances than $d_{c M}$ and $d_{c P}$ for merger to take place.

The results presented here are in agreement with past studies on the merger of both barotropic and baroclinic idealized vortices (Masina and Pinardi 1991, Carton 1992, Valcke and Verron 1997) and are mostly due to the chosen vortex structure in terms of potential vorticity. The novelty of our approach is given by the choice of the high-resolution primitive equations context. This allowed us to investigate the merger of realistic three-dimensional subsurface-intensified lenses and the consequent deformation of their structure; as well as the production of small-scale features.

In particular, we studied the merger of baroclinic, shielded subsurface anticyclones (Morel and McWilliams 1997). The assumption of isolated (shielded) vortex is in general more realistic compared to like-signed-PV vortices (Olson 1980, Paillet et al. 2002).

Merger related effects have also been investigated. We could determine the structure of the final merged vortex for $\mathrm{M}$ and P-Vortices. During and after the merging process, the initial circular-section vortices switch to an elliptic shape as a result of the vortex-vortex interaction. The initial, shielded PV is maintained for several months. Furthermore, the merging process reinforces the vortex, mainly increasing its horizontal extension. These results are also corroborated by observations of merging events in the real ocean. Meddies (corresponding to our idealized M-vortices), as they drift away from the Iberian coasts, go through several merging processes and their aspect ratio progressively decreases, as pointed out in Bashmachnikov et al. (2015).

Besides this, production of long-lived, small-scale features has also been observed. Subsurface, lens-like anticyclones can in fact be generated by the instability of peripheral filaments. Their radii can go down to $10 \mathrm{~km}$ and they can live for several months, in the absence of other larger-scale structures than the merged vortex.

\section{Acknowledgements}

The authors wish to thank Igor Bashmachnikov for fruitful discussions, as well as Gildas Cambon and Nicolas Grima for technical support. This study was funded by Université de Bretagne Occidentale (UBO) and Région Bretagne. 


\section{References}

Armi, L. and Zenk, W., Large Lenses of Highly Saline Mediterranean Water. J. Phys. Oceanogr., 1984, 14, $1560-1576$

Bambrey, R.R., Reinaud, J.N. and Dritschel, D.G., Strong interactions between two corotating quasigeostrophic vortices. J. Fluid Mech., 2007, 592, 117-133.

Barbosa Aguiar, A.C., Peliz, Á. and Carton, X., A census of Meddies in a long-term high-resolution simulation. Progr. Oceanogr., 2013, 116, 80-94.

Bashmachnikov, I. and Carton, X., Surface signature of Mediterranean Water eddies in the Northeastern Atlantic: effect of the upper ocean stratification. Ocean Sci., 2012, 8, 931-943.

Bashmachnikov, I., Neves, F., Calheiros, T. and Carton, X., Properties and pathways of Mediterranean water eddies in the Atlantic. Progr. in Oceanogr., 2015, 137, 149-172.

Carton, X., Hydrodynamical Modeling of Oceanic Vortices. Surv. Geophys., 2001, 22, 179-263.

Carton, X., On the merger of shielded vortices. Europhys. Lett., 1992, 18, 697-703.

Cenedese, C., Adduce, C. and Fratantoni, D.M., Laboratory experiments on mesoscale vortices interacting with two islands. J. Geophys. Res.:Oceans, 2005, 110.

Chelton, D., De Szoeke, R., Schlax, M., El Naggar, K. and Siwertz, N., Geographical Variability of the First Baroclinic Rossby Radius of Deformation. J. Phys. Oceanogr., 1998, 28, 433-459.

Chelton, D.B., Schlax, M.G. and Samelson, R.M., Global observations of nonlinear mesoscale eddies. Prog. Oceanogr., 2011, 91, $167-216$.

Ciani, D., Carton, X., Bashmachnikov, I., Chapron, B. and Perrot, X., Influence of deep vortices on the ocean surface. Interdiscip. j. discontin. nonlinearity complex., 2015, 4, 281-311.

Cushman-Roisin, B. and Beckers, J.M., Introduction to geophysical fluid dynamics: physical and numerical aspects, Vol. 101, 2011 (Academic Press).

Delbende, I., Piton, B. and Rossi, M., Merging of two helical vortices. Eur. J. Mech. B-Fluid, 2015, 49, 363-372.

Dritschel, D.G., The stability and energetics of corotating uniform vortices. J. Fluid Mech., 1985, 157, 95-134.

Dritschel, D.G., A general theory for two-dimensional vortex interactions. J. Fluid Mech., 1995, 293, 269-303.

Dritschel, D.G. and Legras, B., The elliptical model of two-dimensional vortex dynamics. II: Disturbance equations. Phys. Fluids A, 1991, 3, 855-869.

Ebbesmeyer, C.C., Taft, B.A., Mcwilliams, J.C., Shen, C.Y., Riser, S.C., Rossby, H.T., Biscaye, P.E. and Östlund, H.G., Detection, structure, and origin of extreme anomalies in a Western Atlantic oceanographic section. J. Phys. Oceanogr., 1986, 16, 591-612.

Filyushkin, B. and Sokolovskiy, M., Modeling the evolution of intrathermocline lenses in the Atlantic Ocean. J. Mar. Res., 2011, 69, 191-220.

Filyushkin, B., Sokolovskiy, M., Kozhelupova, N. and Vagina, I., Evolution of intrathermocline eddies moving over a submarine hill; in Doklady Earth Sciences, Vol. 441, 2011, pp. 1757-1760.

Kieu, C.Q. and Zhang, D.L., Genesis of Tropical Storm Eugene (2005) from merging vortices associated with ITCZ breakdowns. Part I: Observational and modeling analyses. J. Atmos. Sci., 2008, 65, 3419-3439.

L'Hégaret, P., Carton, X., Ambar, I., Ménesguen, C., Hua, B.L., Chérubin, L., Aguiar, A., Cann, B., Daniault, N. and Serra, N., Evidence of Mediterranean Water dipole collision in the Gulf of Cadiz. J. Geophys. Res.: Oceans, 2014, 119, 5337-5359.

L'Hégaret, P., Etude de la circulation de mésoéchelle et de sorties d'eaux du Golfe Persique dans l'Océan Indien Nord-Ouest. PhD Manuscript, Université de Bretagne Occidentale, 2015.

Masina, S. and Pinardi, N., Merging of barotropic symmetric vortices. A case study for Gulf Stream rings. Nuovo Cimento C, 1991, 14, 539-553.

Meschanov, S. and Shapiro, G., A young Lens of Red Sea Water in the Arabian Sea. Deep Sea Res., 1998, 45, $1-13$.

Molemaker, M.J., McWilliams, J.C. and Dewar, W.K., Submesoscale instability and generation of mesoscale anticyclones near a separation of the California Undercurrent. J. Phys. Oceanogr., 2014.

Morel, Y. and McWilliams, J., Evolution of isolated interior vortices in the ocean. J. Phys. Oceanogr., 1997, 27, 727-748.

Olson, D.B., The physical oceanography of two rings observed by the cyclonic ring experiment. Part II: Dynamics. J. Phys. Oceanogr., 1980, 10, 514-528.

Overman, E.A., Zabusky, N.J. et al., Evolution and merger of isolated vortex structures.. 1981, Technical report, DTIC Document. 
Paillet, J., Le Cann, B., Carton, X., Morel, Y. and Serpette, A., Dynamics and evolution of a northern Meddy. J. Phys. Oceanogr., 2002, 32, 55-79.

Pingree, R. and Le Cann, B., Structure of a Meddy (Bobby 92) southeast of the Azores. Deep Sea Res., 1993, 40, 2077-2103.

Ponte, A.L., Klein, P., Capet, X., Le Traon, P.Y., Chapron, B. and Lherminier, P., Diagnosing surface mixed layer dynamics from high-resolution satellite observations: Numerical insights. J. Phys. Oceanogr., 2013, 43, $1345-1355$.

Reinaud, J.N. and Dritschel, D.G., The critical merger distance between two co-rotating quasi-geostrophic vortices. J. Fluid Mech., 2005, 522, 357-381.

Richardson, P., Bower, A. and Zenk, W., A census of Meddies tracked by floats. Progr. Oceanogr., 2000, 45, 209-250.

Rubino, A., Falcini, F., Zanchettin, D., Bouche, V., Salusti, E., Bensi, M., Riccobene, G., De Bonis, G., Masullo, R., Simeone, F. et al., Abyssal undular vortices in the Eastern Mediterranean basin. Nature, 2012, 3, 834.

Schultz Tokos, K.L., Hinrichsen, H.H. and Zenk, W., Merging and migration of two Meddies. J. Phys. Oceanogr., 1994, 24, 2129-2141.

Senjyu, T., Ishimaru, T., Matsuyama, M. and Koike, Y., High Salinity Lens from the Strait of Hormuz. Offshore Environment of the ROPME Sea Area after the War-Related Oil Spill, Eds. A. Otsuki et al., 1998, pp. 35-48.

Shapiro, G. and Meschanov, S., Distribution and spreading of Red Sea Water and salt lens formation in the northwest Indian Ocean. Deep Sea Res., 1991, 38, 21 - 34.

Shchepetkin, A.F. and McWilliams, J.C., The regional oceanic modeling system (ROMS): a split-explicit, free-surface, topography-following-coordinate oceanic model. Ocean Model., 2005, 9, 347-404.

Sokolovskiy, M.A., Filyushkin, B.N. and Carton, X.J., Dynamics of intrathermocline vortices in a gyre flow over a seamount chain. Ocean Dynam., 2013, 63, 741-760.

Trieling, R., Fuentes, O.V. and van Heijst, G., Interaction of two unequal corotating vortices. Phys. Fluids (1994-present), 2005, 17, 87-103.

Valcke, S. and Verron, J., Interactions of baroclinic isolated vortices: The dominant effect of shielding. J. Phys. Oceanogr., 1997, 27, 524-541.

Vallis, G.K., Atmospheric and Oceanic Fluid Dynamics, 2006 (Cambridge, U.K.: Cambridge University Press).

Verron, J., Hopfinger, E. and McWilliams, J., Sensitivity to initial conditions in the merging of two-layer baroclinic vortices. Phys. Fluids A, 1990, 2, 886-889.

Waugh, D.W., The efficiency of symmetric vortex merger. Phys.Fluids A, 1992, 4, 1745-1758. 